\title{
On Closures of Preimages of Metric Projection Mappings in Hilbert Spaces
}

\author{
Dariusz Zagrodny ${ }^{1}$
}

Received: 12 October 2014 / Accepted: 12 October 2015 / Published online: 22 October 2015

(C) The Author(s) 2015. This article is published with open access at Springerlink.com

\begin{abstract}
The closure of preimages (inverse images) of metric projection mappings to a given set in a Hilbert space are investigated. In particular, some properties of fibers over singletons (level sets or preimages of singletons) of the metric projection are provided. One of them, a sufficient condition for the convergence of minimizing sequence for a giving point, ensures the convergence of a subsequence of minimizing points, thus the limit of the subsequence belongs to the image of the metric projection. Several examples preserving this sufficient condition are provided. It is also shown that the set of points for which the sufficient condition can be applied is dense in the boundary of the preimage of each set from a large class of subsets of the Hilbert space. As an application of obtained properties of preimages we show that if the complement of a nonconvex set is a countable union of preimages of convex closed sets then there is a point such that the value of the metric projection mapping is not a singleton. It is also shown that the Klee result, stating that only convex closed sets can be weakly closed Chebyshev sets, can be obtained for locally weakly closed sets.
\end{abstract}

Keywords Inverse mapping of metric projection - Chebyshev sets · Best approximation · Convexity $\cdot$ Differentiability of the distance function $\cdot$ Concavity of the distance function

Mathematics Subject Classification (2010) Primary 49J52, 41A50 · Secondary 41A65, 52A $40,46 \mathrm{C} 05$

This paper is dedicated to Professor Lionel Thibault to honor great man and great mathematician. Thank You Lionel for the journeys in mathematics which we have gone together.

Dariusz Zagrodny

d.zagrodny@uksw.edu.pl

1 Faculty of Mathematics and Natural Sciences, College of Science, Cardinal Stefan Wyszyński University, Dewajtis 5, Warsaw, Poland 


\section{Introduction}

Let $(X,\|\cdot\|)$ be a real normed vector space. For every nonempty subset $S \subset X$ the distance function from the subset $S$ is denoted by $d_{S}(\cdot)$, that is,

$$
d_{S}(x)=\inf _{u \in S}\|u-x\|, \quad \forall x \in X .
$$

For a given subset $S^{\prime} \subset X$ we put

$$
D_{S^{\prime}}(S):=\left\{x \in X \mid d_{S}(x)=d_{S^{\prime}}(x)\right\}
$$

and define the fiber over $s$ as

$$
D_{S}(S):=\left\{x \in X \mid d_{S}(x)=\|x-s\|\right\} .
$$

Of course $D_{S^{\prime}}(S)=D_{S}(S)$, whenever $S^{\prime}=\{s\}$. Moreover, it follows from the continuity of $d_{S}(\cdot)$ and $d_{S^{\prime}}(\cdot)$ that $D_{S^{\prime}}(S)$ is closed.

The metric projection mapping on $S$ is defined by

$$
P_{S}(x):=\left\{s \in \operatorname{cl} S \mid d_{S}(x)=\|x-s\|\right\},
$$

where "cl" stands for the strong topological closure.

We recall that a set $S$ is said to be Chebyshev if $P_{S}(x)$ reduces to exactly one element for all $x \in X$.

Let us denote the family of weakly closed subsets of $\mathrm{cl}^{\text {weak }} S$ as follows

$$
C \in W(S) \Longleftrightarrow C \text { is weakly closed and } C \subset \mathrm{cl}^{\text {weak }} S,
$$

where "cl weak" stands for the weak closure (the closure with respect to the weak topology).

Let us also observe that that for every $x \in X, s \in \operatorname{cl} S$ we have

$$
(x, s) \in \operatorname{gph} P_{S}(\cdot) \Longleftrightarrow(s, x) \in \operatorname{gph} D_{(\cdot)}(S),
$$

where $\operatorname{gph} P_{S}(\cdot):=\left\{(x, s) \in X \times \operatorname{cl} S \mid s \in P_{S}(x)\right\}$ and gph $D_{(\cdot)}(S):=\{(s, x) \in$ $\left.\operatorname{cl} S \times \mathbb{H} \mid x \in D_{S}(S)\right\}$, thus we can treat $D_{(\cdot)}(S)$ as the inverse mapping to $P_{S}(\cdot)$, that is $D_{(\cdot)}(S)=P_{S}^{-1}(\cdot)$. Let us notice that sets $D_{S^{\prime}}(S)$ and $D_{\left(S^{\prime}\right)}(S)$ can be different, where $D_{S^{\prime}}(S)$ is defined in (1) and $D_{\left(S^{\prime}\right)}(S)=\left\{x \in X \mid \exists s \in S^{\prime}: x \in D_{S}(S)\right\}$ is related to the mapping defined in (2). However, if $S^{\prime} \in W(S), S^{\prime} \subset \operatorname{cl} S$, and $X$ is a reflexive Banach space, then $D_{S^{\prime}}(S)=D_{\left(S^{\prime}\right)}(S)$. Moreover assuming that $S^{\prime}$ is a closed subset of $\operatorname{cl} S$ such that $\operatorname{cl} D_{S^{\prime}}(S)=\operatorname{cl}$ int $D_{S^{\prime}}(S)$, we have $D_{S^{\prime}}(S)=\operatorname{cl} D_{\left(S^{\prime}\right)}(S)$, whenever $P_{S^{\prime}}(\cdot) \neq \varnothing$ on a dense subset of a Banach space (for example in the case of a reflexive locally uniformly convex space, see [28, Theorem 5]). The equalities $D_{S^{\prime}}(S)=D_{\left(S^{\prime}\right)}(S)$ or $D_{S^{\prime}}(S)=\operatorname{cl} D_{\left(S^{\prime}\right)}(S)$, which are valid in several important cases, allows us to investigate the preimages of the metric projection on $S$ using sets $D_{S^{\prime}}(S)$ instead of $P_{S}^{-1}\left(S^{\prime}\right)$ in several important cases. In particular, if $\mathrm{X}$ is a real Hilbert space then $D_{S^{\prime}}(S)=\operatorname{cl} P_{S}^{-1}\left(S^{\prime}\right)$ for every closed subset $S^{\prime}$ of $\operatorname{cl} S$ such that $\operatorname{cl} D_{S^{\prime}}(S)=\operatorname{cl}$ int $D_{S^{\prime}}(S)$. That is the closure of the preimage of a subset $S^{\prime}$ of cl $S$ for the metric projection mapping to a given set $S$ is the set given in (1), whenever $\operatorname{cl} D_{S^{\prime}}(S)=\operatorname{cl}$ int $D_{S^{\prime}}(S)$.

One of the primary questions concerning the best approximation theory is the question about the convergence of a minimizing sequence. In the language of preimages this question can be expressed in the following way: assume that $u \notin \operatorname{cl} S$ and $\left\{s_{i}\right\}_{i \in \mathbb{N}}$ is a sequence of elements of $S$ such that $u \in D_{\left\{s_{1}, s_{2}, \ldots\right\}}(S)$ and $u \notin \bigcup_{i \in \mathbb{N}} D_{s_{i}}(S)$, under which conditions the sequence contains a convergent subsequence? In Section 3 sufficient conditions for the convergence of a minimizing sequence are given, whenever $u$ belongs to the boundary of $D_{S^{\prime}}(S)$ and $S^{\prime}$ has some properties, see Proposition 3.1. It is also checked that desired 
properties of $S^{\prime}$ can be guaranteed in several cases (they are listed in remarks in this section). As a simple consequence of the sufficient condition it is obtained that any "smooth" point $u$ from the boundary of $D_{S}(S)$ has the property that any sequence of "almost" nearest points from $S$ has to converge to the nearest point to $u$, whenever the interior of the fiber is nonempty. In Section 4 results showing the density of "smooth points" in boundaries of some sets are presented. The nonemptiness of a fiber ensures that if the inverse mapping to the projection on $S$ is injective (i.e. the mapping $d \rightrightarrows D_{d}(S), d \in S$ is such that $D_{d_{1}}(S) \cap D_{d_{2}}(S)=\emptyset, d_{1} \neq d_{2}, d_{1}, d_{2} \in S$ ), then $s$ is a supporting point of $S$, see Corollary 5.2 in Section 5. It is also observed that if the inverse mapping to the projection on $S$ is injective then $S$ has no isolated point, see Corollary 5.4 . In Section 7 a result characterizing those cases whenever a converging subsequece does not exist is provided, see Theorem 7.1. In Section 2 basic information is gathered. Moreover, some basic properties of fibers can be found there, for example like: convexity and closedness, see Lemma 2.3; the distance function is Fréchet differentiable on int $D_{S}(S)$, this is a direct consequence of Lemma 2.4.

Properties of the metric projection mapping are useful in investigating sets and differential properties of the distance function from a set. For example, it is known that if the metric projection of a Chebyshev set is continuous then the set is convex, see [1, Corollary page 237] for details in Hilbert spaces and to [34, Theorem 4] in smooth reflexive spaces. The continuity of the metric projection mapping is closely related to the differentiability of the distance function from a set, see [35, page 56] and also [33, 34] for more on the link between the differentablity of the distance function and the continuity of the metric projection mapping. Several aspects of differentiability of the squared distance or the continuity of the metric projection can be also found in $[1-3,5,7,11,13,14,18,19,23,30,36]$. Herein we use the L.P. Vlasov condition given in Section 6, see (22), to preserve the continuity of the metric projection in the case $S$ is locally weakly closed or the complement of $S$ is included in a countable union of preimages of convex closed sets, see Theorems 8.7 and 8.10 in Section 8. Thus the convexity of $S$ is preserved in these cases.

Let us recall that the convexity of Chebyshev sets in Hilbert space is regarded as one of the most important problems of abstract approximation theory: see [24], where it was posed: "However, even in a Hilbert space it remains unknown whether a Chebyshev set must be convex, or, equivalently, whether it must be weakly closed"; see [22, Problem 5] The Possible Convexity of a Chebyshev Set in a Hilbert Space, where a recent review of some achievements in solving the problem is given.

The author would like to thank Prof. M. Turzański for several stimulating discussions during writing of this paper.

\section{Preliminaries}

In this section some properties of subsets of Hilbert space are gathered.

For every real $r>0$ and every $x \in X$ we denote by $B_{X}(x, r)$ (resp. $B_{X}[x, r]$ ) the open (resp. closed) ball centered at $x$ and of radius $r$, the unit sphere of $X$ is denoted by $S_{X}[0,1]:=\{x \in X \mid\|x\|=1\}$, the boundary of a subset $D \subset X$ is denoted by fr $D$, where fr $D:=\operatorname{cl} D \backslash$ int $D$, and "int" stands for the interior of $D$. For given $x, y \in X$ we put $[x, y]:=\{t x+(1-t) y \mid t \in[0,1]\}$ and $] x, y[:=\{t x+(1-t) y \mid t \in] 0,1[\}$

The topological dual space of $X$ is denoted by $X^{*}$, its dual norm by \|\|$_{*}$, that is,

$$
\left\|x^{*}\right\|_{*}:=\sup _{\|u\| \leq 1}\left|\left\langle x^{*}, u\right\rangle\right|=\sup _{\|u\| \leq 1}\left\langle x^{*}, u\right\rangle
$$


where $\langle\cdot, \cdot\rangle$ is the duality pairing between $X$ and $X^{*}$. If $X$ is a Hilbert space with a real inner product (a real Hilbert space), then $\langle x, y\rangle=\frac{1}{4}\left(\|x+y\|^{2}-\|x-y\|^{2}\right.$ ) see for example $[12$, page $25,(1.9)]$. When there is no risk of confusion, we will write $\left\|x^{*}\right\|$ in place of $\left\|x^{*}\right\|_{*}$. The closed unit ball centered at the origin of $X^{*}$ (resp. $\left.X\right)$ is denoted by $\mathbb{B}_{X^{*}}$ (resp. $\left.\mathbb{B}_{X}\right)$.

In the Lemma below it is recalled that the distance function from a set $S$ is a concave function, whenever the complement of the set is convex, see for example [20, Proposition 1, p. 66] and comments following the Proposition.

Lemma 2.1 Let $X$ be a Banach space, $U \subset X$ be a convex open set, $S:=X \backslash U$, and $z \in U$. Then for every $x, y \in \mathbb{U}, \alpha>0, \beta>0$ such that $\alpha+\beta=1, z=\alpha x+\beta y$ we have

$$
\alpha d_{S}(x)+\beta d_{S}(y) \leq d_{S}(z) .
$$

Below the convexity of sets $D_{s}(S)$ is shown, whenever $X$ is a real Hilbert space. Let us start with a simple property of the norm.

Lemma 2.2 Let $\mathbb{H}$ be a Hilbert space, and let $s, u, x, y \in \mathbb{H}, \alpha>0, \beta>0$ be given such that $\alpha+\beta=1, z=\alpha x+\beta y$. If

$$
\|x-s\| \leq\|x-u\| \text { and }\|y-s\| \leq\|y-u\|,
$$

then

$$
\|z-s\| \leq\|z-u\| .
$$

Proof For $x, y, s, u \in \mathbb{H}$ such that the inequalities in (6) are valid we have

$$
\begin{aligned}
& -2\langle x, s\rangle+\|s\|^{2} \leq-2\langle x, u\rangle+\|u\|^{2}, \\
& -2\langle y, s\rangle+\|s\|^{2} \leq-2\langle y, u\rangle+\|u\|^{2},
\end{aligned}
$$

thus

$\alpha\left(-2\langle x, s\rangle+\|s\|^{2}\right)+\beta\left(-2\langle y, s\rangle+\|s\|^{2}\right) \leq \alpha\left(-2\langle x, u\rangle+\|u\|^{2}\right)+\beta\left(-2\langle y, u\rangle+\|u\|^{2}\right)$,

which implies

$$
\|z-s\|^{2}=\|z\|^{2}-2\langle z, s\rangle+\|s\|^{2} \leq\|z\|^{2}-2\langle z, u\rangle+\|u\|^{2}=\|z-u\|^{2} .
$$

Lemma 2.3 Let $\mathbb{H}$ be a real Hilbert space, $S \subset \mathbb{H}$ and $s \in \operatorname{cl} S$ be given. The set $D_{s}(S)$ is convex and closed.

Proof of the convexity: Let $x, y \in D_{s}(S), \alpha>0, \beta>0, \alpha+\beta=1, z=\alpha x+\beta y$, $u \in S$. If

$$
\begin{aligned}
& d_{S}(x)=\|x-s\| \leq\|x-u\|, \\
& d_{S}(y)=\|y-s\| \leq\|y-u\|,
\end{aligned}
$$


then it follows from Lemma 2.2 that

$$
d_{S}(z) \leq\|z-s\| \leq\|z-u\|,
$$

thus

$$
d_{S}(z) \leq\|z-s\| \leq \inf _{u \in S}\|z-u\|=d_{S}(z)
$$

of closedness: Let $\left\{d_{i}\right\}_{i \in \mathbb{N}}$ be a sequence of elements of subset $D_{s}(S)$ such, that $\lim _{i \longrightarrow \infty} d_{i}=d$. We have $d_{S}\left(d_{i}\right)=\left\|d_{i}-s\right\|$ for every $i \in \mathbb{N}$, so by the continuity of $d_{S}(\cdot)$ and $\|\cdot-s\|$ we get $d_{S}(d)=\|d-s\|$, which implies $d \in D_{s}(S)$.

Let us assume that $\bar{s} \in \operatorname{cl} S, u \in D_{\bar{S}}(S) \backslash\{\bar{s}\}$. It is of interest that each point $u_{\rho} \in D_{\bar{s}}(S)$ has the following property

$$
\left\|u_{\rho}-s_{i}\right\| \longrightarrow d_{S}\left(u_{\rho}\right) \Longrightarrow s_{i} \longrightarrow \bar{s},
$$

where $u_{\rho}:=\rho u+(1-\rho) \bar{s}, \rho \in\left[0,1\left[\right.\right.$ and $\left\{s_{i}\right\}_{i \in \mathbb{N}}$ is a sequence of elements from $S$, this result was given in [38, Lemma 1.5]. In other words we have the continuity of the metric projection mapping at each $u_{\rho} \in D_{\bar{s}}(S)$, thus we have the Fréchet differentiability of the distance function at each $u_{\rho} \in D_{\bar{S}}(S) \backslash(\operatorname{cl} S \cup\{u\})$, see [13, Theorem 3.1]. This property can be also found in the proof of [23, Proposition 4.1]. For the sake of the reader's convenience we provide the result with a proof.

Lemma 2.4 Let $\mathbb{H}$ be a real Hilbert space, and $S \subset H$ be a nonempty subset, $\bar{s} \in \operatorname{cl} S$ and $u \in D_{\bar{s}}(S)$ with $d_{S}(u)>0$ be given. Then for all $\rho \in[0,1[$ we have

$$
0=\lim _{\mu \downarrow 0} \sup \left\{\|\bar{s}-s\| \mid s \in S \text { and }\left\|u_{\rho}-s\right\| \leq \sqrt{d_{S}^{2}\left(u_{\rho}\right)+\mu^{2}}\right\},
$$

where $u_{\rho}:=\rho u+(1-\rho) \bar{s}$.

Proof Fix $\rho \in[0,1[$. Suppose

$$
\delta:=2^{-1} \lim _{\mu \downarrow 0} \sup \left\{\|\bar{s}-s\| \mid s \in S \text { and }\left\|u_{\rho}-s\right\| \leq \sqrt{d_{S}^{2}\left(u_{\rho}\right)+\mu^{2}}\right\}>0 .
$$

Take a sequence $\left\{\mu_{i}\right\}_{i \in \mathbb{N}}$ such that $\left.\mu_{i} \in\right] 0,1\left[\right.$ for all $i \in \mathbb{N}$ and $\mu_{i} \downarrow 0$, and a sequence $\left\{s_{i}\right\}_{i \in \mathbb{N}}$ elements of $S$ satisfying

$$
\left\|u_{\rho}-s_{i}\right\| \leq \sqrt{d_{S}^{2}\left(u_{\rho}\right)+\mu_{i}^{2}} \text { and }\left\|s_{i}-\bar{s}\right\| \geq \delta
$$

for all $i \in \mathbb{N}$. Observe that for each $i \in \mathbb{N}$ we have

$$
\sqrt{\left\|u_{\rho}-\bar{s}\right\|^{2}+\mu_{i}^{2}} \geq\left\|u_{\rho}-s_{i}\right\|
$$

and

$$
\left\|u-s_{i}\right\| \geq\|u-\bar{s}\|,
$$

so we obtain $2\left\langle u-\bar{s}, \bar{s}-s_{i}\right\rangle+\left\|\bar{s}-s_{i}\right\|^{2} \geq 0$ and consequently

$$
\mu_{i}^{2} \geq 2\left\langle u_{\rho}-\bar{s}, \bar{s}-s_{i}\right\rangle+\left\|\bar{s}-s_{i}\right\|^{2} \geq(1-\rho)\left\|\bar{s}-s_{i}\right\|^{2} \geq(1-\rho) \delta^{2},
$$

hence $0 \geq 1-\rho$, which is impossible. 
Corollary 2.5 Let $\mathbb{H}$ be a real Hilbert space, $S \subset \mathbb{H}$ be a nonempty subset and $S^{\prime} \subset \operatorname{cl} S$ be a given closed nonempty subset such that int $D_{S^{\prime}}(S) \neq \emptyset$; and $u \in D_{S^{\prime}}(S) \backslash$ int $D_{S^{\prime}}(S), u \notin$ $S^{\prime}, s \in S^{\prime} \cap P_{S}(u)$ are such that $] s, u\left[\cap\right.$ int $D_{S^{\prime}}(S) \neq \emptyset$. Then $u+t(u-s) \notin D_{s}(S)$ for all $t \in] 0, \infty[$.

Proof Let us suppose the contrary, namely $u+t(u-s) \in D_{S}(S)$ for some $\left.t \in\right] 0, \infty[$. Take any sequence $\left\{u_{i}\right\}_{i \in \mathbb{N}}$ of elements of $\mathbb{H} \backslash D_{S^{\prime}}(S)$ such that $u_{i} \longrightarrow u$. There is a sequence $\left\{s_{i}\right\}_{i \in \mathbb{N}}$ in $S \backslash\left\{S^{\prime}\right\}$ such that

$$
d_{S^{\prime}}\left(u_{i}\right)>\left\|u_{i}-s_{i}\right\|
$$

for every $i \in \mathbb{N}$. Observe that for $\rho:=\frac{1}{1+t}$ we have

$$
\begin{aligned}
& u=\rho(u+t(u-s))+(1-\rho) s \\
& \quad \text { and } \lim _{i \longrightarrow \infty}\left\|u-s_{i}\right\|=\lim _{i \longrightarrow \infty}\left\|u_{i}-s_{i}\right\|=\lim _{i \longrightarrow \infty} d_{S^{\prime}}\left(u_{i}\right)=d_{S^{\prime}}(u)=d_{S}(u),
\end{aligned}
$$

thus by Lemma 2.4 the sequence $\left\{s_{i}\right\}_{i \in \mathbb{N}}$ is converging to $s$. Notice that since $S^{\prime}$ is closed, so $d_{S^{\prime}}\left(s_{i}\right)>0$, hence $s_{i} \notin \operatorname{cl} D_{S^{\prime}}(S)$. Take $b_{i} \in\left(\operatorname{clint} D_{S^{\prime}}(S) \backslash \operatorname{int} D_{S^{\prime}}(S)\right) \cap\left[s_{i}, s_{i}+\alpha\left(u_{i}-\right.\right.$ $\left.\left.s_{i}\right)\right]$, where $\alpha u+(1-\alpha) s \in$ int $\left.D_{S^{\prime}}(S), \alpha \in\right] 0,1[$. We have

$$
\begin{aligned}
\left\|u_{i}-s_{i}\right\|<d_{S^{\prime}}\left(u_{i}\right) \leq d_{S^{\prime}}\left(b_{i}\right)+\left\|u_{i}-b_{i}\right\|= & d_{S}\left(b_{i}\right)+\left\|u_{i}-b_{i}\right\| \\
& \leq\left\|b_{i}-s_{i}\right\|+\left\|u_{i}-b_{i}\right\|=\left\|u_{i}-s_{i}\right\|
\end{aligned}
$$

for every $i \in \mathbb{N}$, which is impossible.

If a directional derivative of the distance function is equal to 1 for a unit vector at some point outside the considered set, then any minimizing sequence is converging, this is a simple consequence of [15, Proposition 2.3]. Below it is shown that the diameter of the set of minimizing points is related to the value of the directional derivative of the distance function from the set.

Lemma 2.6 Let $\mathbb{H}$ be a real Hilbert space, and $S \subset H$ be a nonempty subset, $u \notin \operatorname{cl} S$, $h \in S_{\mathbb{H}}[0,1]$ be given. If

$$
\lim _{t \downarrow 0} \frac{d_{S}(u+t h)-d_{S}(u)}{t}=\gamma,
$$

then for $\bar{s}:=u-d_{S}(u) h$ we have

$$
\lim _{\mu \downarrow 0} \sup \left\{\|\bar{s}-s\| \mid s \in S \text { and }\|u-s\| \leq \sqrt{d_{S}^{2}(u)+\mu^{2}}\right\} \leq \sqrt{2(1-\gamma)} d_{S}(u) .
$$

Proof Suppose that

$$
\sqrt{2(1-\gamma)} d_{S}(u)<\lim _{\mu \downarrow 0} \sup \left\{\|\bar{s}-s\| \mid s \in S \text { and }\|u-s\| \leq \sqrt{d_{S}^{2}(u)+\mu^{2}}\right\} .
$$

Take any

$$
\delta \in] \sqrt{2(1-\gamma)} d_{S}(u), \lim _{\mu \downarrow 0} \sup \left\{\|\bar{s}-s\| \mid s \in S \text { and }\|u-s\| \leq \sqrt{d_{S}^{2}(u)+\mu^{2}}\right\}[,
$$

$\epsilon>0,\left\{\mu_{i}\right\}_{i \in \mathbb{N}}$ in ]0,1[ with $\mu_{i} \downarrow 0$, and a sequence $\left\{s_{i}\right\}_{i \in \mathbb{N}}$ of elements of $S$ satisfying

$$
\left\|u-s_{i}\right\| \leq \sqrt{d_{S}^{2}(u)+\mu_{i}^{2}} \text { and }\left\|s_{i}-\bar{s}\right\|^{2} \geq \delta^{2}>d_{S}(u)\left(2(1-\gamma) d_{S}(u)+\epsilon\right)
$$


for every $i \in \mathbb{N}$. Observe that for every $i \in \mathbb{N}$ we have

$$
\|u-\bar{s}\|^{2}+\mu_{i}^{2} \geq\left\|u-s_{i}\right\|^{2},
$$

and consequently

$$
\mu_{i}^{2} \geq 2\left\langle u-\bar{s}, \bar{s}-s_{i}\right\rangle+\left\|\bar{s}-s_{i}\right\|^{2}=2 d_{s}(u)\left\langle h, \bar{s}-s_{i}\right\rangle+\left\|\bar{s}-s_{i}\right\|^{2} .
$$

The condition $\lim _{t \downarrow 0} \frac{d_{s}(u+t h)-d_{S}(u)}{t}=\gamma$ ensures that

$$
\left\|u+\mu_{i} h-s_{i}\right\|^{2}-\|u-\bar{s}\|^{2} \geq 2 \mu_{i}\left(\gamma-\frac{\epsilon}{2 d_{S}(u)}\right) d_{S}(u)
$$

for $i \in \mathbb{N}$ large enough. Hence

$$
\begin{gathered}
\mu_{i} 2\left(\gamma-\frac{\epsilon}{2 d_{S}(u)}\right) d_{S}(u) \leq\left\|u+\mu_{i} h-s_{i}\right\|^{2}-\|u-\bar{s}\|^{2}=\left\|u-s_{i}\right\|^{2}+2 \mu_{i}\left\langle h, u-s_{i}\right\rangle+\mu_{i}^{2} \\
-\|u-\bar{s}\|^{2} \leq\|u-\bar{s}\|^{2}+\mu_{i}^{2}+2 \mu_{i}\left\langle h, u-s_{i}\right\rangle+\mu_{i}^{2}-\|u-\bar{s}\|^{2}=2 \mu_{i}^{2} \\
+2 \frac{\mu_{i}}{d_{S}(u)}\left(d_{S}^{2}(u)+\left\langle u-\bar{s}, \bar{s}-s_{i}\right\rangle\right)=2 \mu_{i}^{2}+2 \frac{\mu_{i}}{d_{S}(u)}\left(d_{S}^{2}(u)+\left\langle u-\bar{s}, \bar{s}-s_{i}\right\rangle\right. \\
\left.+\frac{1}{2}\left\|\bar{s}-s_{i}\right\|^{2}-\frac{1}{2}\left\|\bar{s}-s_{i}\right\|^{2}\right) \leq 2 \mu_{i}^{2}+2 \frac{\mu_{i}}{d_{S}(u)}\left(d_{S}^{2}(u)+\frac{1}{2} \mu_{i}^{2}-\frac{1}{2} \delta^{2}\right),
\end{gathered}
$$

thus

$$
\mu_{i} 2\left(\gamma-\frac{\epsilon}{2 d_{S}(u)}\right) d_{S}(u) \leq 2 \mu_{i}^{2}+2 \frac{\mu_{i}}{d_{S}(u)}\left(d_{S}^{2}(u)+\frac{1}{2} \mu_{i}^{2}-\frac{1}{2} \delta^{2}\right)
$$

for $i \in N$ large enough, so

$$
\delta^{2} \leq 2(1-\gamma) d_{S}^{2}(u)+\epsilon d_{S}(u)
$$

which by the choice of $\delta$ and $\epsilon$ is impossible, thus (8) is valid.

In fact we have equality in (8). The reverse inequality is obtained in Lemma 8.1. Let us also mention that we have the strong convergence of any minimizing sequence, whenever $\gamma=1$.

It is known that norms of elements of a weakly converging sequence can be far from the norm of its weak limit. This is a drawback of the weak convergence which sometimes can be overcome, for example whenever the Kadec-Klee property is valid. Below a condition allowing to estimate from below the norm of the weak limit of a sequence by norms of its elements is provided.

Lemma 2.7 Let $\mathbb{H}$ be a real Hilbert space, $X, Y \subset \mathbb{H}$ be closed subspaces such that $\mathbb{H}=$ $X+Y, X$ has a finite dimension and $\langle x, y\rangle=0$ for every $x \in X, y \in Y$; and $\epsilon>0$ be given. Then for all sequences $\left\{x_{i}\right\}_{i \in \mathbb{N}}$ in $\mathbb{H},\left\{y_{i}\right\}_{i \in \mathbb{N}}$ in $\mathbb{H}$ such that $x_{i} \stackrel{\text { weak }}{\longrightarrow} x^{*}, y_{i} \stackrel{\text { weak }}{\longrightarrow} y^{*}$ and $\lim \sup _{i \rightarrow \infty}\left\|P_{Y}\left(x_{i}-y_{i}\right)\right\| \leq \epsilon \limsup _{i \rightarrow \infty}\left\|P_{Y}\left(y_{i}\right)\right\| \leq \epsilon$ and $2 \epsilon<\lim \sup _{i \rightarrow \infty}\left\|x_{i}\right\|$ we have

$$
\left\|x^{*}\right\| \geq \limsup _{i \rightarrow \infty} \sqrt{\left\|x_{i}\right\|^{2}-4 \epsilon^{2}}
$$

where “weak $\longrightarrow$ "stands for the weak convergence.

Proof Assume that $\limsup _{i \rightarrow \infty}\left\|x_{i}\right\|=\lim _{i \rightarrow \infty}\left\|x_{i}\right\|$, if not then a proper subsequence can be chosen. Observe that by the local compactness of $X$ we can choose a subsequence 
such that $\left\{P_{X}\left(x_{i_{k}}\right)\right\}_{k \in \mathbb{N}}$ is converging, again, without loss of generality we may assume that $\left\{P_{X}\left(x_{i}\right)\right\}_{i \in \mathbb{N}}$ converges. We have

$$
\begin{aligned}
&\left\|x^{*}\right\| \geq\left\|P_{X}\left(x^{*}\right)\right\|=\lim _{i \longrightarrow \infty}\left\|P_{X}\left(x_{i}\right)\right\| \\
&=\sqrt{\lim _{i \rightarrow \infty}\left(\left\|P_{X}\left(x_{i}\right)\right\|^{2}+\left\|P_{Y}\left(x_{i}\right)\right\|^{2}-\left\|P_{Y}\left(x_{i}\right)\right\|^{2}\right)} \\
&=\sqrt{\lim _{i \rightarrow \infty}\left(\left\|x_{i}\right\|^{2}-\left\|P_{Y}\left(x_{i}\right)\right\|^{2}\right)} \\
& \geq \sqrt{\limsup _{i \longrightarrow \infty}\left(\left\|x_{i}\right\|^{2}-\left(\left\|P_{Y}\left(y_{i}\right)\right\|+\left\|P_{Y}\left(x_{i}\right)-P_{Y}\left(y_{i}\right)\right\|\right)^{2}\right)} \\
& \geq \sqrt{\limsup _{i \longrightarrow \infty}\left\|x_{i}\right\|^{2}-4 \epsilon^{2}} .
\end{aligned}
$$

We finish this section with an observation, which is intuitively obvious, namely if $D$ is a set, $u \in$ fr $D$ is a "smooth" boundary point, then moving from $u$ along a tangent direction to $D$ we are sufficiently close to the set $D$. For the sake of the reader's convenience we provide this property in the Hilbert space setting.

Lemma 2.8 Let $\mathbb{H}$ be a real Hilbert space with, $D \subset \mathbb{H}$ be a subset with nonempty interior. Assume that for $u \in \mathrm{cl}$ int $D \backslash$ int $D$ there is $x \in$ int $D$ such that $d_{\mathbb{H} \backslash \text { int } D}(x)=\|u-x\|$. Then there is $u^{*} \in S_{\mathbb{H}}[0,1]$ such that

$$
\lim _{t \longrightarrow 0}|t|^{-1} \sup _{z \in S_{\mathbb{H}}[0,1],\left\langle z, u^{*}\right\rangle=0} d_{D}(u+t z)=0 .
$$

Proof Put $u^{*}:=\|u-x\|^{-1}(u-x)$. For every $z \in S_{\mathbb{H}}[0,1]$ for which $\left\langle z, u^{*}\right\rangle=0$ and every $t \in \mathbb{R}$ we have

$$
\begin{aligned}
& d_{D}(u+t z) \leq d_{\mathbb{B}_{\mathbb{H}}[x,\|u-x\|]}(u+t z) \\
& \leq\left\|u+t z-\left(x+\frac{\|u-x\|}{\|u+t z-x\|}(u+t z-x)\right)\right\| \\
& =\|u+t z-x\|\left(1-\frac{\|u-x\|}{\|u+t z-x\|}\right)=\|u+t z-x\|-\|u-x\| \\
& \quad=\sqrt{\|u-x\|^{2}+t^{2}}-\sqrt{\|u-x\|^{2}} \leq \frac{t^{2}}{2\|u-x\|},
\end{aligned}
$$

which implies

$$
\lim _{t \longrightarrow 0}|t|^{-1} \sup _{z \in S_{\mathbb{H}}[0,1],\langle z, u-x\rangle=0} d_{D}(u+t z)=0 .
$$

\section{Points from the Set of Points of Approximate Compactness}

Let $S \subset \mathbb{H}$ and $u \notin S$ be given. We say that $u$ is a point of approximate compactness of $S$ if each minimizing sequence, that is $s_{i} \in S$ and $\left\|u-s_{i}\right\| \longrightarrow d_{S}(u)$, contains a 
convergent subsequence to the nearest point to $u$. The set of the all points of approximate compactness of $S$ we denote $A C(S)$, we refer to [2, page 1131] for more information on the notion. Following [2, see page 1131] we say that $S$ is approximately compact whenever $\mathbb{H} \backslash S=A C(S)$, see [2] for historical references.

It was observed in (7) that for each $u \in \operatorname{fr} D_{s}(S), \rho \in\left[0,1\left[\right.\right.$ and each sequence $\left\{s_{i}\right\}_{i \in \mathbb{N}}$ of elements of $S$ such that $\left\|\rho u+(1-\rho) s-s_{i}\right\| \longrightarrow d_{S}(\rho u+(1-\rho) s)$, the sequence $\left\{s_{i}\right\}_{i \in \mathbb{N}}$ has to converge to $s$, so $\rho u+(1-\rho) s \in A C(S)$. It is also interesting that each "smooth" point $u \in \operatorname{fr} D_{s}(S)$ is an element of $A C(S)$, whenever int $D_{s}(S) \neq \emptyset$, this is a consequence of the result below. In fact, in Proposition 3.1 a more general characterization of some points from $A C(S)$ is given. This characterization allows us to preserve the continuity of the metric projection mapping at those "smooth" points for which the metric projection is a singleton. Of course, the question is when the assumptions of Proposition 3.1 are fulfilled. In Remarks $3.2,3.3,3.4,3.5$ some results answering the question are presented.

Proposition 3.1 Let $\mathbb{H}$ be a real Hilbert space, $S \subset \mathbb{H}$ be a nonempty subset, $S^{\prime} \subset \mathbb{H}$, $\bar{s} \in S^{\prime} \cap \operatorname{cl} S$ with $D_{\bar{s}}(S) \neq \mathbb{H}$ be given. Assume that for a given $u \in(\mathbb{H} \backslash S) \cap A C\left(S^{\prime}\right)$ we have $\bar{s} \in P_{S}(u) \cap S^{\prime}, P_{S^{\prime}}(u)=\{\bar{s}\}$ and there is $u^{*} \in S_{\mathbb{H}}[0,1]$ such that

$$
\lim _{t \downarrow 0} t^{-1} \sup _{z \in S_{\mathbb{H}}[0,1],\left\langle z, u^{*}\right\rangle=0} d_{D_{S^{\prime}}(S)}(u+t z)=0 .
$$

Assume that for a given sequence $\left\{t_{i}\right\}_{i \in \mathbb{N}}, t_{i}>0, t_{i} \longrightarrow 0$ there is a function $o:[0, \infty[\longrightarrow$ $\left[0, \infty\left[\right.\right.$ such that $o(0)=0, \lim _{i \rightarrow \infty} \frac{o\left(t_{i}\right)}{t_{i}}=0$ with the property such that for every $i \in \mathbb{N}$ and $z \in S_{\mathbb{H}}[0,1]$ with $\left\langle z, u^{*}\right\rangle=0$ there exists $d \in D_{S^{\prime}}(S) \backslash\{u\}$ such that $\left\|u+t_{i} z-d\right\| \leq$ $o\left(t_{i}\right)$, and there exists $s^{\prime} \in S^{\prime}$ such that $\left\|d-s^{\prime}\right\| \leq \sqrt{d_{S}^{2}(d)+2 o\left(t_{i}\right)}$ and $\left\|s^{\prime}-u\right\| \geq$ $\|\bar{s}-u\|$. Then each sequence $\left\{s_{i}\right\}_{i \in \mathbb{N}}$ of elements of $S$ such that $\left\|s_{i}-u\right\| \longrightarrow\|\bar{s}-u\|$ has a convergent subsequence, say $s_{i_{k}} \longrightarrow s \in \operatorname{cl} S$, moreover $d_{S}(u)=\|s-u\|=\|\bar{s}-u\|$ and $s-\bar{s} \in\left[-2\|\bar{s}-u\| u^{*}, 2\|\bar{s}-u\| u^{*}\right]$.

Proof Put

$$
p(t):=\max \left\{1,4 d_{S}^{2}(u)\right\}\left(\max \left\{2 o(t), \sup _{z \in S_{\mathbb{H}}[0,1],\left\langle z, u^{*}\right\rangle=0} d_{D_{S^{\prime}}(S)}(u+t z)\right\}+t^{2}\right),
$$

for every $t \geq 0$. Assume that $\left\{s_{i}\right\}_{i \in \mathbb{N}}$ is a sequence of elements from $S$ such that $\left\|s_{i}-u\right\| \longrightarrow$ $\|\bar{s}-u\|$. Take $M>0$ such that $\left\|s_{i}-\bar{s}\right\|<M$ for all $i \in \mathbb{N}$. Let us suppose that

$$
\limsup _{i \longrightarrow \infty} d_{\left[-M u^{*}, M u^{*}\right]}\left(s_{i}-\bar{s}\right)>0 .
$$

We may assume that for some $\delta>0$ and all $i \in \mathbb{N}$ we have

$$
d_{\left[-M u^{*}, M u^{*}\right]}\left(s_{i}-\bar{s}\right)>\delta \text { and } t_{i}^{2}>\left\|s_{i}-u\right\|^{2}-\|\bar{s}-u\|^{2} .
$$

If not then we can take a proper subsequence. For each $i \in \mathbb{N}$ choose $z_{i} \in S_{\mathbb{H}}[0,1]$ satisfying $\left\langle z_{i}, u^{*}\right\rangle=0,\left\langle s_{i}-\bar{s}, z_{i}\right\rangle>\delta$, and take $d_{i} \in D_{S^{\prime}}(S)$ satisfying $\left\|d_{i}-u-t_{i} z_{i}\right\| \leq o\left(t_{i}\right)$. There is a sequence $\left\{\bar{s}_{i}\right\}_{i \in \mathbb{N}}$ such that $\bar{s}_{i} \in S^{\prime}$ and $\left\|d_{i}-\bar{s}_{i}\right\| \leq \sqrt{d_{S}^{2}\left(d_{i}\right)+2 o\left(t_{i}\right)}$, and $\left\|\bar{s}_{i}-u\right\| \geq\|\bar{s}-u\|$ for every $i \in \mathbb{N}$. For $i$ large enough have also

$$
\begin{gathered}
t_{i}^{2}+\|\bar{s}-u\|^{2}-2 t_{i}\left\langle s_{i}-u, z_{i}\right\rangle+t_{i}^{2}+7 p\left(t_{i}\right) \geq\left\|s_{i}-u\right\|^{2}-2 t_{i}\left\langle s_{i}-u, z_{i}\right\rangle+t_{i}^{2} \\
\quad+7 p\left(t_{i}\right) \geq\left\|s_{i}-u-t_{i} z_{i}\right\|^{2}+7 p\left(t_{i}\right) \geq\left\|s_{i}-d_{i}\right\|^{2}+4 p\left(t_{i}\right) \geq\left\|d_{i}-\bar{s}_{i}\right\|^{2}+3 p\left(t_{i}\right) \\
\geq\left\|u+t_{i} z_{i}-\bar{s}_{i}\right\|^{2}=\left\|\bar{s}_{i}-u\right\|^{2}+2 t_{i}\left\langle u-\bar{s}_{i}, z_{i}\right\rangle+t_{i}^{2} \geq\|\bar{s}-u\|^{2}+2 t_{i}\left\langle u-\bar{s}_{i}, z_{i}\right\rangle+t_{i}^{2},
\end{gathered}
$$


consequently

$$
\frac{t_{i}^{2}+7 p\left(t_{i}\right)}{t_{i}} \geq\left\langle s_{i}-\bar{s}, z_{i}\right\rangle+\left\langle\bar{s}-\bar{s}_{i}, z_{i}\right\rangle \geq \delta+\left\langle s_{i}-\bar{s}_{i}, z_{i}\right\rangle
$$

for every $i \in \mathbb{N}$ large enough. We have

$$
\lim _{i \longrightarrow \infty}\left\|u-\bar{s}_{i}\right\|=\lim _{i \rightarrow \infty}\left\|d_{i}-\bar{s}_{i}\right\|=\lim _{i \longrightarrow \infty} d_{S^{\prime}}\left(d_{i}\right)=d_{S^{\prime}}(u) .
$$

Since $u \in A C\left(S^{\prime}\right)$ we are able to find a subsequence of the sequence $\left\{\bar{s}_{i}\right\}_{i \in \mathbb{N}}$ which is convergent, say $\bar{s}_{i_{k}} \longrightarrow \bar{s}^{\prime} \in \operatorname{cl} S^{\prime} \cap P_{S^{\prime}}(u)$ (keep in mind the assumption $P_{S^{\prime}}(u)=\{\bar{s}\}$ ), which implies $\bar{s}_{i_{k}} \longrightarrow \bar{s}$ and

$$
0=\lim _{k \longrightarrow \infty} \frac{t_{i_{k}}^{2}+7 p\left(t_{i_{k}}\right)}{t_{i_{k}}} \geq \delta>0,
$$

a contradiction, so

$$
\lim _{i \longrightarrow \infty} d_{\left[-M u^{*}, M u^{*}\right]}\left(s_{i}-\bar{s}\right)=0 .
$$

The compactness of the segment $\left[-M u^{*}, M u^{*}\right]$ ensures the existence of a convergent subsequence $\left\{s_{i_{k}}\right\}_{k \in \mathbb{N}}$, say $s_{i_{k}} \longrightarrow s \in \mathrm{cl} S$. Of course we have also $\|u-s\|=\|u-\bar{s}\|$ and $s-\bar{s} \in\left[-M u^{*}, M u^{*}\right]$, hence since $\|s-\bar{s}\| \leq 2\|u-\bar{s}\|$ we get $s-\bar{s} \in\left[-2\|\bar{s}-u\| u^{*}, 2 \| \bar{s}-\right.$ $\left.u \| u^{*}\right]$.

In order to show that assumptions of Proposition 3.1 are not difficult to verify we present several examples below. In the first example $S^{\prime}$ is assumed to be convex. The convexity of $S^{\prime}$ gives the equality $\mathbb{H} \backslash S^{\prime}=A C\left(S^{\prime}\right)$, so $S^{\prime}$ is aproximately compact.

Remark 3.2 Let $\mathbb{H}$ be a real Hilbert space, $S \subset \mathbb{H}$ be a nonempty subset and $S^{\prime} \subset \operatorname{cl} S$ be a nonempty convex closed subset. Assume that for a given $u \notin S$ we have

$$
\bar{s} \in P_{S}(u) \cap S^{\prime},
$$

and there is $u^{*} \in S_{\mathbb{H}}[0,1]$ such that

$$
\lim _{t \downarrow 0} t^{-1} \sup _{z \in S_{\mathbb{H}}[0,1],\left\langle z, u^{*}\right\rangle=0} d_{D_{S^{\prime}}(S)}(u+t z)=0 .
$$

Put $o(t):=4\left(\sup _{z \in S_{\text {开 }}[0,1],\left\langle z, u^{*}\right\rangle=0} d_{D_{S^{\prime}}(S)}(u+t z)+t^{2}\right)$ and fix a sequence $\left\{t_{i}\right\}_{i \in \mathbb{N}}$ such that $t_{i}>0, t_{i} \longrightarrow 0$. Then for every $i \in \mathbb{N}$ and $z \in S_{\mathbb{H}}$ such that $\left\langle z, u^{*}\right\rangle=0$ there exists $d \in D_{S^{\prime}}(S) \backslash\{u\}$ such that $\left\|u+t_{i} z-d\right\| \leq o\left(t_{i}\right)$, and there exists $s^{\prime} \in S^{\prime}$ such that $\left\|d-s^{\prime}\right\| \leq \sqrt{d_{S}^{2}(d)+2 o\left(t_{i}\right)}$ and $\left\|s^{\prime}-u\right\| \geq\|\bar{s}-u\|$. Moreover $P_{S^{\prime}}(u)=\{\bar{s}\}$. Thus the assumptions of Proposition 3.1 are satisfied.

Proof Take any $z \in S_{\mathbb{H}}[0,1]$ satisfying $\left\langle z, u^{*}\right\rangle=0$ and fix $i \in \mathbb{N}$. Because of (10) we are able to choose $d \in D_{S^{\prime}}(S) \backslash\{u\}$ such that $\left\|u+t_{i} z-d\right\| \leq 2^{-1} o\left(t_{i}\right)$. There is a unique $s^{\prime} \in S^{\prime}$, see [16, Lemma 6.2.1, page 205] for example, such that $d_{S}(d)=d_{S^{\prime}}(d)=\left\|d-s^{\prime}\right\|$. Additionally $P_{S^{\prime}}(u)=\{\bar{s}\}$, since $S^{\prime}$ is closed and convex.

Approximately compact sets have the property that any Chebyshev set which is approximately compact has to be convex, see [2]. In Section 8 a local version of this result is presented, see (11) and Theorem 8.7 below. 
Remark 3.3 Let $\mathbb{H}$ be a real Hilbert space, $S \subset \mathbb{H}$ be a nonempty subset, $u \notin S, S^{\prime} \subset \operatorname{cl} S$ be given and $\bar{s} \in S^{\prime}$. Assume that

$$
P_{S}(u) \cap \operatorname{cl} S^{\prime}=\{\bar{s}\} \text { and }\left(\left\|s_{i}^{\prime}-u\right\| \longrightarrow d_{S^{\prime}}(u) \text { and } s_{i}^{\prime \text { weak }} \longrightarrow s \in \mathbb{H} \Longrightarrow s \in \operatorname{cl} S^{\prime}\right)
$$

for every sequence $\left\{s_{i}^{\prime}\right\}_{i \in \mathbb{N}}$ in $S^{\prime}$, and that there is $u^{*} \in S_{\mathbb{H}}[0,1]$ for which

$$
\lim _{t \downarrow 0} t^{-1} \sup _{z \in S_{\mathbb{H}}[0,1],\left\langle z, u^{*}\right\rangle=0} d_{D_{S^{\prime}}(S)}(u+t z)=0 .
$$

Put $o(t):=4\left(\sup _{z \in S_{\mathbb{H}}[0,1],\left\langle z, u^{*}\right\rangle=0} d_{D_{S^{\prime}}(S)}(u+t z)+t^{2}\right)$ and fix a sequence $\left\{t_{i}\right\}_{i \in \mathbb{N}}$ such that $t_{i}>0, t_{i} \longrightarrow 0$. Then for every $i \in \mathbb{N}$ and $z \in S_{\mathbb{H}}$ such that $\left\langle z, u^{*}\right\rangle=0$ there exists $d \in D_{S^{\prime}}(S) \backslash\{u\}$ such that $\left\|u+t_{i} z-d\right\| \leq o\left(t_{i}\right)$, and there exists $s^{\prime} \in S^{\prime}$ such that $\left\|d-s^{\prime}\right\| \leq \sqrt{d_{S}^{2}(d)+2 o\left(t_{i}\right)}$ and $\left\|s^{\prime}-u\right\| \geq\|\bar{s}-u\|$. Moreover $P_{S^{\prime}}(u)=\{\bar{s}\}$. Thus the assumptions of Proposition 3.1 are satisfied.

Proof In order to show that $u \in A C\left(S^{\prime}\right)$ let us take a minimizing sequence $\left\{s_{i}^{\prime}\right\}_{i \in \mathbb{N}}$ in $S^{\prime}$ such that

$$
\left\|s_{i}^{\prime}-u\right\| \longrightarrow d_{S^{\prime}}(u) .
$$

It is known that from each minimizing sequence we are able to choose a weakly convergent subsequence, see [12, Theorem 3.139] for example, say $\left\{s_{i_{k}}^{\prime}\right\}_{k \in \mathbb{N}}$ such that $s_{i_{k}}^{\prime} \stackrel{\text { weak }}{\longrightarrow} s \in \mathbb{H}$. It follows from (11) that $s \in \mathrm{cl} S^{\prime}$, so $s \in P_{S}(u)$ and $\left\|s_{i_{k}}^{\prime}-u\right\| \longrightarrow d_{S^{\prime}}(u)=\|s-u\|$, hence $s_{i_{k}}^{\prime} \longrightarrow s$ (the Kadec-Klee property of the norm in the Hilbert space setting is used to get the strong convergence). Observe also that we have the inclusion $P_{S^{\prime}}(u) \subset P_{S}(u)$, which by (11) gives

$$
\bar{s} \in P_{S^{\prime}}(u) \cap \operatorname{cl} S^{\prime} \subset P_{S}(u) \cap \operatorname{cl} S^{\prime}=\{\bar{s}\},
$$

hence $P_{S^{\prime}}(u)=\{\bar{s}\}$.

Take any $z \in S_{\mathbb{H}}[0,1]$ satisfying $\left\langle z, u^{*}\right\rangle=0$ and fix $i \in \mathbb{N}$. Because of (12) we are able to choose $d \in D_{S^{\prime}}(S) \backslash\{u\}$ such that $\left\|u+t_{i} z-d\right\| \leq 2^{-1} o\left(t_{i}\right)$. There is $s^{\prime} \in S^{\prime}$ such that $\left\|d-s^{\prime}\right\| \leq \sqrt{d_{S^{\prime}}^{2}(d)+2 o\left(t_{i}\right)}=\sqrt{d_{S}^{2}(d)+2 o\left(t_{i}\right)}$ and $\left\|s^{\prime}-u\right\| \geq\|\bar{s}-u\|$.

It is known that $P_{S}(u)$ is a singleton, whenever the distance function $d_{S}(\cdot)$ is Fréchet differentiable at $u \notin \mathrm{cl} S$, see also [13, Theorem 2.4], a part of the proof of the Remark below can be also used to get it. We use the idea behind the result to get a local sequential weak closedness of $S$, that is we prove that (11) holds true, whenever $d_{S}(\cdot)$ is Fréchet differentiable at $u \notin \mathrm{cl} S$.

Remark 3.4 Let $\mathbb{H}$ be a real Hilbert space, $S \subset \mathbb{H}$ be a nonempty subset, $u \notin S, S^{\prime} \subset$ cl $S$ be given and $\bar{s} \in S^{\prime}$. Suppose that the Fréchet derivative of the distance function $d_{S^{\prime}}(\cdot)$ exists at $u$, that is

$$
\exists x^{*} \in \mathbb{H}: \lim _{t \downarrow 0} \sup _{h \in S_{\mathbb{H}}[0,1]} \frac{d_{S^{\prime}}(u+t h)-d_{S^{\prime}}(u)-\left\langle x^{*}, t h\right\rangle}{t}=0,
$$

then $P_{S^{\prime}}(u) \neq \emptyset$. Moreover, if $d_{S}(u)=d_{S^{\prime}}(u)=\|u-\bar{s}\|$ then $\{\bar{s}\}=P_{S}(u) \cap S^{\prime}$ and

$$
\left\|s_{i}-u\right\| \longrightarrow d_{S}(u) \Longrightarrow s_{i} \longrightarrow \bar{s},
$$

for every sequence $\left\{s_{i}\right\}_{i \in \mathbb{N}}$ in $S^{\prime}$. If additionally there is $u^{*} \in S_{\mathbb{H}}[0,1]$ such that

$$
\lim _{t \downarrow 0} t^{-1} \sup _{z \in S_{\mathbb{H}}[0,1],\left\langle z, u^{*}\right\rangle=0} d_{D_{S^{\prime}}(S)}(u+t z)=0,
$$


then for $o(t):=4\left(\sup _{z \in S_{\mathbb{H}}[0,1],\left\langle z, u^{*}\right\rangle=0} d_{D_{S^{\prime}}(S)}(u+t z)+t^{2}\right)$ and any sequence $\left\{t_{i}\right\}_{i \in \mathbb{N}}$ such that $t_{i}>0, t_{i} \longrightarrow 0$ we have that for every $i \in \mathbb{N}$ and $z \in S_{\mathbb{H}}$ such that $\left\langle z, u^{*}\right\rangle=0$ there exists $d \in D_{S^{\prime}}(S) \backslash\{u\}$ such that $\left\|u+t_{i} z-d\right\| \leq o\left(t_{i}\right)$, and there exists $s^{\prime} \in S^{\prime}$ such that $\left\|d-s^{\prime}\right\| \leq \sqrt{d_{S}^{2}(d)+2 o\left(t_{i}\right)}$ and $\left\|s^{\prime}-u\right\| \geq\|\bar{s}-u\|$. Moreover $P_{S^{\prime}}(u)=\{\bar{s}\}$. Thus the assumptions of Proposition 3.1 are satisfied.

Proof It follows from Lemma 2.6 that $P_{S^{\prime}}(u) \neq \emptyset$, it is enough to apply Lemma 2.6 for $h:=x^{*}$ and observe that $\|h\|=1$, see [23, Remark 3.1 and Theorem 3.2 or Theorem 4.2] for example. Moreover, if $\left\{s_{i}\right\}_{i \in \mathbb{N}}$ is a sequence of elements from $S^{\prime}$ such that $\left\|s_{i}-u\right\| \longrightarrow$ $d_{S}(u)=d_{S^{\prime}}(u)$, then the strong convergence $s_{i} \longrightarrow \bar{s}$ is again a consequence of Lemma 2,6 .

Observe also that we have the inclusion $P_{S^{\prime}}(u) \subset P_{S}(u)$, which implies

$$
\bar{s} \in P_{S^{\prime}}(u) \cap \operatorname{cl} S^{\prime} \subset P_{S}(u) \cap \operatorname{cl} S^{\prime}=\{\bar{s}\},
$$

hence $P_{S^{\prime}}(u)=\{\bar{s}\}$.

If additionally there is $u^{*} \in S_{\mathbb{H}}[0,1]$ such that

$$
\sup _{z \in S_{\mathbb{H}}[0,1],\left\langle z, u^{*}\right\rangle=0} d_{D_{S^{\prime}}(S)}(u+t z)=o(t),
$$

then take any $z \in S_{\mathbb{H}}[0,1]$ satisfying $\left\langle z, u^{*}\right\rangle=0$. As in the proof of Remark 3.3 we are able to choose $d \in D_{S^{\prime}}(S) \backslash\{u\}$ such that $\left\|u+t_{i} z-d\right\| \leq 2^{-1} o\left(t_{i}\right)$. There is $s^{\prime} \in S^{\prime}$ such that $\left\|d-s^{\prime}\right\| \leq \sqrt{d_{S^{\prime}}^{2}(d)+2 o\left(t_{i}\right)}=\sqrt{d_{S}^{2}(d)+2 o\left(t_{i}\right)}$ and $\left\|s^{\prime}-u\right\| \geq\|\bar{s}-u\|$.

Remark 3.5 Let $\mathbb{H}$ be a real Hilbert space, $S \subset \mathbb{H}$ be a nonempty subset, $\bar{s} \in S, u \notin S$, $S^{\prime} \subset \operatorname{cl} S$ be given such that $d_{S}(u)=d_{S^{\prime}}(u)=\|u-\bar{s}\|$ and

$$
\forall s^{\prime} \in S^{\prime},\left\langle u-\bar{s}, s^{\prime}-\bar{s}\right\rangle \leq 0 .
$$

Then $\{\bar{s}\}=P_{S}(u) \cap S^{\prime}$ and

$$
\left\|s_{i}-u\right\| \longrightarrow d_{S}(u) \Longrightarrow s_{i} \longrightarrow \bar{s},
$$

for every sequence $\left\{s_{i}\right\}_{i \in \mathbb{N}}$ in $S^{\prime}$. If additionally there is $u^{*} \in S_{\mathbb{H}}[0,1]$ such that

$$
\lim _{t \downarrow 0} t^{-1} \sup _{z \in S_{\mathbb{H}}[0,1],\left\langle z, u^{*}\right\rangle=0} d_{D_{S^{\prime}}(S)}(u+t z)=0,
$$

then for $o(t):=4\left(\sup _{z \in S_{\mathbb{H}}[0,1],\left\langle z, u^{*}\right\rangle=0} d_{D_{S^{\prime}}(S)}(u+t z)+t^{2}\right)$ and any sequence $\left\{t_{i}\right\}_{i \in \mathbb{N}}$ such that $t_{i}>0, t_{i} \longrightarrow 0$ we have that for every $i \in \mathbb{N}$ and $z \in S_{\mathbb{H}}$ such that $\left\langle z, u^{*}\right\rangle=0$ there exists $d \in D_{S^{\prime}}(S) \backslash\{u\}$ such that $\left\|u+t_{i} z-d\right\| \leq o\left(t_{i}\right)$, and there exists $s^{\prime} \in S^{\prime}$ such that $\left\|d-s^{\prime}\right\| \leq \sqrt{d_{S}^{2}(d)+2 o\left(t_{i}\right)}$ and $\left\|s^{\prime}-u\right\| \geq\|\bar{s}-u\|$. Moreover $P_{S^{\prime}}(u)=\{\bar{s}\}$. Thus the assumptions of Proposition 3.1 are satisfied.

Proof Put $x^{*}:=d_{S^{\prime}}^{-1}(u)(u-\bar{s})$. If

$$
\lim _{t \downarrow 0} \frac{d_{S^{\prime}}\left(u+t x^{*}\right)-d_{S^{\prime}}(u)}{t}=1,
$$

then it follows from Lemma 2.6 that condition (15) holds true, hence $\{\bar{s}\}=P_{S}(u) \cap S^{\prime}$. 
Let us exclude the case

$$
\lim _{t \downarrow 0} \frac{d_{S^{\prime}}\left(u+t x^{*}\right)-d_{S^{\prime}}(u)}{t}<1-\mu
$$

for some $\mu>0$. Take sequences $\left\{s_{i}^{\prime}\right\}_{i \in \mathbb{N}}$ in $S^{\prime}$ and $\left\{t_{i}^{\prime}\right\}_{i \in \mathbb{N}}$ in $] 0,1\left[, t_{i}^{\prime} \downarrow 0\right.$ for which $\left\|u+t_{i}^{\prime} x^{*}-s_{i}^{\prime}\right\|^{2} \leq t_{i}^{\prime 2}+d_{S^{\prime}}^{2}\left(u+t_{i}^{\prime} x^{*}\right)$ and

$$
\left\|u+t_{i}^{\prime} x^{*}-s_{i}^{\prime}\right\|^{2}-\|u-\bar{s}\|^{2} \leq 2 t_{i}^{\prime}(1-\mu) d_{S^{\prime}}(u)+t_{i}^{\prime 2}
$$

for every $i \in \mathbb{N}$. Since $\left\|u+t_{i}^{\prime} x^{*}-\bar{s}\right\|=d_{S^{\prime}}(u)+t_{i}^{\prime}$, using (14) we get

$$
\begin{aligned}
& 2 t_{i}^{\prime}(1-\mu) d_{S^{\prime}}(u)+t_{i}^{\prime 2} \geq\left\|u+t_{i}^{\prime} x^{*}-s_{i}^{\prime}\right\|^{2}-\|u-\bar{s}\|^{2}=\left\|u+t_{i}^{\prime} x^{*}-\bar{s}\right\|^{2} \\
&+2\left\langle u+t_{i}^{\prime} x^{*}-\bar{s}, \bar{s}-s_{i}^{\prime}\right\rangle+\left\|\bar{s}-s_{i}^{\prime}\right\|^{2}-\|u-\bar{s}\|^{2} \geq\left\|u+t_{i}^{\prime} x^{*}-\bar{s}\right\|^{2} \\
&+\left\|\bar{s}-s_{i}^{\prime}\right\|^{2}-\|u-\bar{s}\|^{2} \geq\left(d_{S^{\prime}}(u)+t_{i}^{\prime}\right)^{2}-d_{S^{\prime}}^{2}(u),
\end{aligned}
$$

for every $i \in \mathbb{N}$. Hence

$$
2(1-\mu) d_{S^{\prime}}(u) \geq \lim _{i \rightarrow \infty} \frac{\left(d_{S^{\prime}}(u)+t_{i}^{\prime}\right)^{2}-d_{S^{\prime}}^{2}(u)}{t_{i}^{\prime}}=2 d_{S^{\prime}}(u),
$$

a contradiction. In order to finish the proof it is enough to repeat the reasoning from Remark 3.4 .

There are two natural examples for $S^{\prime}$ to fulfill condition (14), namely if $S^{\prime} \subset a+\{h \in$ $\left.\mathbb{H} \mid\left\langle x^{*}, h\right\rangle=0\right\}$ for some $a \in \mathbb{H}, x^{*} \in S_{\mathbb{H}}[0,1]$ and $u-\bar{s}=\alpha x^{*}$ for some $\alpha>0$, then it is obvious that (14) is satisfied. Condition (14) is also satisfied, whenever $S^{\prime}$ is convex and $d_{S}(u)=d_{S^{\prime}}(u)=\|u-\bar{s}\|$.

\section{Density Properties}

Let $S, S^{\prime}$ be a subsets of a real Hilbert space. "Smooth points" of the boundary of $D_{S^{\prime}}(S)$ have the "convergence property" as it is observed in the previous section. Of course the state of the art in variational analysis allows us to expect that "smooth points" of the boundary are dense in the boundary. It seems that using ideas from [27], see also [38], we can get this kind of density in every Banach space with the norm Fréchet differentiable and having the Kadec-Klee property. Since we are interested only in the Hilbert space setting we provide such a result in a Hilbert space. The key tool to get it is the Borwein-Preiss Variational Principle, we use a form of the Variational Principle from [6, Theorem 2.5.2].

Definition 4.1 Let $(X, d)$ be a complete metric space. We say that a continuous function $\rho: X \times X \rightarrow[0,+\infty]$ is a gauge-type function provided that

(i) $\rho(x, x)=0$ for all $x \in X$,

(ii) for any $\varepsilon>0$ there exists $\delta>0$ such that

$$
\rho(y, z) \leq \delta \text { implies } d(y, z)<\varepsilon \text { for all } y, z \in X .
$$


Theorem 4.2 Let $(X, d)$ be a complete metric space and let $f: X \rightarrow \mathbb{R} \cup\{+\infty\}$ be a lsc function bounded from below. Suppose that $\rho$ is a gauge-type function and $\left(\delta_{i}\right)_{i=0}^{\infty}$ is a sequence of positive numbers, and suppose that $\epsilon>0$ and $x_{0} \in X$ satisfy

$$
f\left(x_{0}\right) \leq \inf _{z \in X} f(z)+\epsilon .
$$

There exist $x \in X$ and a sequence $\left(x_{i}\right)_{i=1}^{\infty} \subset X$ such that

(i) $\rho\left(x_{o}, x\right) \leq \frac{\epsilon}{\delta_{0}}, \rho\left(x_{i}, x\right) \leq \frac{\epsilon}{2^{i} \delta_{0}}$,

(ii) $f(x)+\sum_{i=1}^{\infty} \delta_{i} \rho\left(x, x_{i}\right) \leq f\left(x_{0}\right)$,

(iii) $\quad f(y)+\sum_{i=1}^{\infty} \delta_{i} \rho\left(y, x_{i}\right)>f(x)+\sum_{i=1}^{\infty} \delta_{i} \rho\left(x, x_{i}\right)$ for all $y \in X \backslash\{x\}$

Below we present a result which is a consequence of [28, Theorem 4]. It says that for every given point $u \notin S$ there is a point $u^{\prime}$ close to the given point such that $P_{S}\left(u^{\prime}\right) \neq \emptyset$. For the sake of the reader's convenience a proof is provided.

Proposition 4.3 Let $\mathbb{H}$ be a real Hilbert space, $U \subset \mathbb{H}$ be an open nonempty subset such that $\mathbb{H} \backslash U \neq \emptyset$, and $w \in U$ be fixed. For every $\mu>0$ there are $x \in U \cap \mathbb{B}_{\mathbb{H}}[w, \mu]$, $s \in$ fr $U$ such that $d_{\mathbb{H} \backslash U}(x)=\|x-s\|$ and any sequence $\left\{w_{i}\right\}_{i \in \mathbb{N}}$ in $\mathbb{H} \backslash U$ such that $\left\|w_{i}-x\right\| \longrightarrow d_{\mathbb{H} \backslash U}(x)$ has a convergent subsequence (with respect to the norm topology), say $\left\{w_{i_{n}}\right\}_{n \in \mathbb{N}}$ for which $s=\lim _{n} \longrightarrow \infty w_{i_{n}}$.

Proof Let us fix $\mu>0$. Take any $\left.\delta \in] 0, \frac{\min \left\{\mu, d_{\mathbb{H} \backslash U}(w)\right\}}{4}\right]$ and put $K:=\mathbb{H} \backslash U$,

$$
\begin{aligned}
f(z):=d_{K}(z)+\frac{16^{2} d_{K}\left(x_{0}\right)}{\delta^{2}}\left\|z-x_{0}\right\|^{2}, \rho(z, y):=\|z-y\|^{2}, & \\
\epsilon & :=f\left(x_{0}\right), \delta_{0}:=1, x_{0}:=w, \delta_{i}:=\frac{d_{K}\left(x_{0}\right)}{4^{i}} \text { for } i \geq 1 .
\end{aligned}
$$

It follows from Theorem 4.2 that there are $x \in B_{\mathbb{H}}\left[x_{0}, \frac{\delta}{16}\right]$ (keep in mind that applying (ii) we have the inequality $\left.d_{K}(x)+\frac{16^{2} d_{K}\left(x_{0}\right)}{\delta^{2}}\left\|x-x_{0}\right\|^{2} \leq d_{K}\left(x_{o}\right)\right)$, so $x \in U$, and there are $x^{*} \in \mathbb{H}$ and $M>0$ (apply (iii)) such that

$$
\forall y \in \mathbb{H}, d_{K}(y)-d_{K}(x)-\left\langle x^{*}, y-x\right\rangle \geq-M\|y-x\|^{2},
$$

which forces $\left\|x^{*}\right\| \leq 1$. Let $\left\{s_{i}\right\}_{n \in \mathbb{N}}$ be a sequence in $K$ such that $\left\|s_{i}-x\right\| \longrightarrow d_{K}(x)$, $s_{i} \stackrel{\text { weak }}{\longrightarrow} s$, where " $\stackrel{\text { weak }}{\longrightarrow}$ " stands for the weak convergence. By (16) we have

$$
\left\|x+t_{i}\left(s_{i}-x\right)-s_{i}\right\|-\left\|x-s_{i}\right\|-\left\langle x^{*}, t_{i}\left(s_{i}-x\right)\right\rangle \geq-2 M t_{i}^{2}\left\|x-s_{i}\right\|^{2},
$$

for a proper choice of $t_{i}>0, t_{i} \longrightarrow 0$ and $i$ large enough, hence

$$
-\left\|x-s_{i}\right\|-\left\langle x^{*},\left(s_{i}-x\right)\right\rangle \geq-2 M t_{i}\left\|x-s_{i}\right\|^{2} .
$$

It follows from (18) and the weak lower-semicontinuity of $\|x-\cdot\|$ that

$$
\begin{aligned}
\liminf _{i \rightarrow \infty}\left\|x-s_{i}\right\| \geq\|x-s\| \geq-\left\langle x^{*},(s-x)\right\rangle=\lim _{i \rightarrow \infty} & -\left\langle x^{*},\left(s_{i}-x\right)\right\rangle \\
& \geq \limsup _{i \rightarrow \infty}\left\|x-s_{i}\right\| \geq\|x-s\|
\end{aligned}
$$

thus $\left\|s_{i}-x\right\| \longrightarrow\|s-x\|$ and $s_{i} \longrightarrow s$, since $s_{i} \stackrel{\text { weak }}{\longrightarrow} s$, in particular $s \in K, d_{K}(x)=\|s-x\|$ and $x^{*}=d_{K}^{-1}(x)(x-s)$. It is easy to observe that $s \in K \cap \operatorname{cl} U$, so $s \in \operatorname{cl} U \backslash U$. 
Let us recall that $x \in B_{\mathbb{H}}\left[w, \frac{\delta}{16}\right]$, which implies the first part of statement. The choice of a convergent subsequence, which is demanded in the second part of the statement, is guaranteed by the above reasoning. In order to see it, let us fix a sequence $\left\{w_{i}\right\}_{i \in \mathbb{N}}$ in $K$ such that $\left\|w_{i}-x\right\| \longrightarrow d_{K}(x)$. Now take any weakly convergent subsequence $\left\{w_{i_{n}}\right\}_{n \in \mathbb{N}} \subset$ $\left\{w_{i}\right\}_{i \in \mathbb{N}}$. Repeating the reasoning, which is presented above to get the strong convergence of the sequence $\left\{s_{i}\right\}_{i \in \mathbb{N}}$, we get the strong convergence of $\left\{w_{i_{n}}\right\}_{n \in \mathbb{N}}$ to $s$ (keep in mind that $\left.x^{*}=d_{K}^{-1}(x)(x-s)=d_{K}^{-1}(x)\left(x-\lim _{n \longrightarrow \infty} w_{i_{n}}\right)\right)$.

Corollary 4.4 Let $\mathbb{H}$ be a real Hilbert space, $U \subset \mathbb{H}$ be a convex open set, $S:=\mathbb{H} \backslash U \neq \emptyset$, and $S^{\prime} \subset S$ be a closed and convex subset. Then $D_{S^{\prime}}(S)$ is convex.

Proof It follows from Lemma 2.1 that (5) holds true. If $x, y \in D_{S^{\prime}}(S)$ then the convexity of $S^{\prime}$ yields

$$
d_{S}(z) \leq d_{S^{\prime}}(z) \leq \alpha d_{S^{\prime}}(x)+\beta d_{S^{\prime}}(y)=\alpha d_{S}(x)+\beta d_{S}(y) \leq d_{S}(z),
$$

which implies $d_{S^{\prime}}(z)=d_{S}(z)$, where $z=\alpha x+\beta y$.

A convex set has the property that the closure of its interior recovers the closure of the set, whenever the interior is nonempty. It is interesting that the set of supporting points is dense in the boundary of a convex set with nonempty interior, this result was due to BishopPhelps and it was an answer to an open problem by V. Klee. Of course the class of sets having this property is larger than the class of convex sets with nonempty interior. Below it is shown that the set of points from the boundary of some set, belonging to the boundary of a ball contained in the set, is dense in the boundary of the set, whenever the considered set belongs to the class.

Proposition 4.5 Let $\mathbb{H}$ be a real Hilbert space, $U \subset \mathbb{H}$ be an open nonempty subset, and $w \in \operatorname{cl} U \backslash U$ be fixed. For every $\mu>0$ there are $u \in(\operatorname{cl} U \backslash U) \cap \mathbb{B}_{\mathbb{H}}[w, \mu], x \in U$ such that $d_{\mathbb{H} \backslash U}(x)=\|u-x\|$ and any sequence $\left\{w_{i}\right\}_{i \in \mathbb{N}}$ in $\mathbb{H} \backslash U$ such that $\left\|w_{i}-x\right\| \longrightarrow d_{\mathbb{H} \backslash U}(x)$ has a convergent subsequence (with respect to the norm topology), say $\left\{w_{i_{n}}\right\}_{n \in \mathbb{N}} \subset\left\{w_{i}\right\}_{i \in \mathbb{N}}$ for which $d_{\mathbb{H} \backslash U}(x)=\left\|x-\lim _{n \longrightarrow \infty} w_{i_{n}}\right\|$.

Proof Let us fix $\mu>0, d \in U \cap B_{\mathbb{H}}\left[w, \frac{\mu}{4}\right]$. We have $d_{\mathbb{H} \backslash U}(d)>0$. Take any $\delta \in$ ]0, $\left.\frac{\min \left\{\mu, d_{\mathbb{H} \backslash U}(d)\right\}}{4}\right]$ and put $K:=\mathbb{H} \backslash U$. It follows from Proposition 4.3 that there are $x \in$ $B_{\mathbb{H}}\left[d, \frac{\delta}{16}\right], u \in \operatorname{cl} U \backslash U$ such that $d_{K}(x)=\|u-x\|$ and if a sequence $\left\{w_{i}\right\}_{i \in \mathbb{N}}$ in $\mathbb{H} \backslash U$ is such that $\left\|w_{i}-x\right\| \longrightarrow d_{K}(x)$ then it has a converging subsequence (with respect to the norm topology), say $\left\{w_{i_{n}}\right\}_{n \in \mathbb{N}} \subset\left\{w_{i}\right\}_{i \in \mathbb{N}}$ for which $d_{\mathbb{H} \backslash U}(x)=\left\|x-\lim _{n} \longrightarrow \infty w_{i_{n}}\right\|$, $u=\lim _{n \longrightarrow \infty} w_{i_{n}}$. It is easy to observe that $u \in K \cap \operatorname{cl} U$, so $u \in \operatorname{cl} U \backslash U$. Let us recall that $x \in B_{\mathbb{H}}\left[d, \frac{\delta}{16}\right], d \in B_{\mathbb{H}}\left[w, \frac{\mu}{4}\right]$, thus $u \in B_{\mathbb{H}}[w, \mu]$, which implies the statement.

Remark 4.6 In the Hilbert space setting, Proposition 4.5 may be used to retrieve Lau's results [28, Theorem 4], [27] or those concerning the differentiability of lower semicontinuous functions which were obtained in J.M. Borwein, S.P. Fitzpatrick and J. R. Giles [5]. For this purpose it is enough to construct a new Hilbert space $\mathcal{H}:=\mathbb{H} \times \mathbb{R}$ and for a lower semicontinuous function such that int $\operatorname{dom} f \neq \varnothing$ apply Proposition 4.5 together with a result concerning the differentiability of the Moreau envelope, for example see [23, Theorem 3.5], to the open set $U:=\{(x, r) \in \mathcal{H} \mid f(x)>r\}$. This is not the aim of this paper, so it is left 
to the interested reader. Let us also notice that int $\operatorname{cl} U=U$, whenever $f$ is a continuous function, so the set $D:=\operatorname{cl} U$ has the property $\operatorname{cl} D=\operatorname{cl}$ int $D$.

Corollary 4.7 Let $\mathbb{H}$ be a real Hilbert space. Let $S \subset \mathbb{H}$ be a nonempty subset, $u \notin \operatorname{cl} S, \bar{s} \in$ $\operatorname{cl} S$ be given such that $\|u-\bar{s}\|=d_{S}(u)$; and $\epsilon>0$ be fixed. Then int $D_{\mathbb{B}_{\mathbb{H}}[\bar{s}, \epsilon] \cap S}(S) \neq \emptyset$, $u \in \mathrm{cl}$ int $D_{\mathbb{B}_{\mathbb{H}}[\bar{s}, \epsilon] \cap S}(S)$ and if there is $u^{\prime} \in\left(\operatorname{cl}\right.$ int $D_{\mathbb{B}_{\mathbb{H}}[\bar{s}, \epsilon] \cap S}(S) \backslash$ int $\left.D_{\mathbb{B}_{\mathbb{H}}[\bar{s}, \epsilon] \cap S}(S)\right)$ such that $d_{\mathbb{H} \backslash \backslash \text { int }} D_{\mathbb{B}_{\mathbb{H}}[\bar{s}, \epsilon] \cap S}(S)=\left\|u^{\prime}-x\right\|$ and the Fréchet derivative of the distance function $d_{S}(\cdot)$ exists at $u^{\prime}$, then $P_{S}\left(u^{\prime}\right)$ is a singleton and $\left\|s^{\prime}-\bar{s}\right\|=\epsilon$, where $s^{\prime} \in P_{S}\left(u^{\prime}\right)$.

Proof It follows from Lemma 2.4 that $(1-\rho) u+\rho \bar{s} \in \operatorname{int} D_{\mathbb{B}_{\mathbb{H}}[\bar{s}, \epsilon] \cap S}(S)$ for all $\rho \in] 0,1\left[\right.$. Assume that $u^{\prime} \in\left(\operatorname{clint} D_{\mathbb{B}_{\mathbb{H}}[\bar{s}, \epsilon] \cap S}(S) \backslash \operatorname{int} D_{\mathbb{B}_{\mathbb{H}}[\bar{s}, \epsilon] \cap S}(S)\right)$ is such that $d_{\mathbb{H} \backslash \text { int } D_{\mathbb{B}_{\mathbb{H}}[\bar{s}, \epsilon] \cap S}(S)}(x)=\left\|u^{\prime}-x\right\|$ and the Fréchet derivative of the distance function $d_{S}(\cdot)$ exists at $u^{\prime}$. Using Remark 3.4 we get $\left\|u^{\prime}-s^{\prime}\right\|=d_{S}\left(u^{\prime}\right)$ for some $s^{\prime} \in \mathbb{B}_{\mathbb{H}}[\bar{s}, \epsilon] \cap S$. If $\left\|s^{\prime}-\bar{s}\right\|<\epsilon$, then it follows from Remark 3.4 that there is $d \in\left(S \backslash\left\{s^{\prime}\right\}\right) \cap P_{S}\left(u^{\prime}\right)$, keep in mind that $u^{\prime} \notin$ int $D_{\mathbb{B}_{\mathbb{H}}[\bar{s}, \epsilon] \cap S}(S)$, so there are $u_{i} \in \mathbb{H}$ and $s_{i} \in S \backslash \mathbb{B}_{\mathbb{H}}[\bar{s}, \epsilon]$ such that $u_{i} \longrightarrow u,\left\|s_{i}-u_{i}\right\| \longrightarrow d_{S}\left(u^{\prime}\right), s_{i} \longrightarrow d$. However, the differentiability of the distance function yields that $P_{S}\left(u^{\prime}\right)$ is singleton, so $d=s^{\prime}$ a contradiction.

Below we provide another example of a possibly non-convex set having the following property

$$
\text { cl } D=\operatorname{clint} D .
$$

If a set has the property above, then it follows from Proposition 4.5 that there is a dense subset of fr $D$, say $F \subset$ fr $D$, such that for every $u \in F$ there is $x \in \operatorname{int} D$ for which $d_{\mathbb{H} \backslash \text { int } D}(u)=\|u-x\|$.

Lemma 4.8 Let $\mathbb{H}$ be a real Hilbert space. Let $S \subset \mathbb{H}$ be a nonempty subset, $S^{\prime} \subset \mathbb{H}$, $\bar{s} \in S^{\prime} \cap \operatorname{cl} S$. Assume that $u \in D_{\bar{s}}(S)$ and there is $\epsilon>0$ for which

$$
\mathbb{B}_{\mathbb{H}}[\bar{s}, \epsilon] \cap\left(S \backslash S^{\prime}\right)=\emptyset,
$$

then $u \in \operatorname{cl}$ int $D_{S^{\prime}}(S)$.

Proof Let us fix any $\rho \in\left[0,1\left[\right.\right.$. Put $u_{\rho}:=\rho u+(1-\rho) \bar{s}$. Let us prove that $u_{\rho} \in \operatorname{int} D_{S^{\prime}}(S)$. If there is a sequence $\left\{u_{i}\right\}_{i \in \mathbb{N}}$ in $\mathbb{H} \backslash D_{S^{\prime}}(S)$ such that $u_{i} \longrightarrow u_{\rho}$, then for every $i \in \mathbb{N}$ put

$$
S_{i}:=\left\{s \in S \mid\left\|s-u_{i}\right\|<\frac{d_{S}\left(u_{i}\right)+d_{S^{\prime}}\left(u_{i}\right)}{2}\right\} .
$$

There is a sequence $\left\{s_{i}\right\}_{i \in \mathbb{N}}$ in $S \backslash S^{\prime}$ such that $\left\|u_{i}-s_{i}\right\| \longrightarrow d_{S}\left(u_{\rho}\right), s_{i} \in S_{i}$ for every $i \in \mathbb{N}$. It follows from Lemma 2.4 that $s_{i} \longrightarrow \bar{s}$. Hence by (19) we obtain $s_{i} \in S^{\prime} \cap \mathbb{B}_{\mathbb{H}}[\bar{s}, \epsilon]$ for $i \in \mathbb{N}$ large enough, which contradicts the choice of the sequence $\left\{s_{i}\right\}_{i \in \mathbb{N}}$. Thus $u_{\rho} \in$ int $D_{S^{\prime}}(S)$ and consequently $u \in \operatorname{clint} D_{S^{\prime}}(S)$.

\section{Sets for which there are Points at which the Metric Projection is not a Singleton}

In this section several results ensuring, for a giving set, the existence of points at which the metric projection is not a singleton are presented. 
Corollary 5.1 Let $\mathbb{H}$ be a real Hilbert space, $S \subset \mathbb{H}$ be a nonempty subset and $S^{\prime} \subset \operatorname{cl} S$ be a given closed convex nonempty subset such that int $D_{S^{\prime}}(S) \neq \emptyset$; and $u \in \operatorname{cl}$ int $D_{S^{\prime}}(S) \backslash$ int $D_{S^{\prime}}(S), u \notin S^{\prime}, s \in S^{\prime} \cap P_{S}(u), u+t(u-s) \notin D_{s}(S)$ for every $\left.t \in\right] 0, \infty$ [. If there is $x \in$ int $D_{S^{\prime}}(S)$ for which $d_{\mathbb{H} \backslash \backslash \text { int } D_{S^{\prime}}(S)}(x)=\|u-x\|$ and $\langle u-s, u-x\rangle \neq 0$, then there exists $d \in \operatorname{cl} S, d \neq s$ such that $u \in D_{d}(S)$.

Proof Let us notice that if $u+t_{0}(u-s) \in D_{S^{\prime}}(S)$ for some $\left.t_{0} \in\right] 0, \infty\left[\right.$, then $u+2^{-1} t_{0}(u-$ $s) \in D_{s}(S)$, since the convexity of $S^{\prime}$, but this contradicts to $u+t(u-s) \notin D_{s}(S)$ for every $t \in] 0, \infty\left[\right.$. Hence, there are sequences $\left\{\epsilon_{i}\right\}_{i \in \mathbb{N}}$ in $] 0, \infty\left[,\left\{s_{i}\right\}_{i \in \mathbb{N}}\right.$ in $S \backslash\left\{S^{\prime}\right\}$ such that $\lim _{i \longrightarrow \infty} \epsilon_{i}=0, u+\epsilon_{i}\|u-s\|^{-1}(u-s) \notin D_{s}(S)$, and

$$
d_{S^{\prime}}\left(u+\epsilon_{i}(u-s)\right)>\left\|u+\epsilon_{i}(u-s)-s_{i}\right\|
$$

for every $i \in \mathbb{N}$. By Proposition 3.1 (see Remark 3.2) and Lemma 2.8 the sequence $\left\{s_{i}\right\}_{i \in \mathbb{N}}$ has a convergent subsequence, say to $d \in \operatorname{cl} S$, and consequently $u \in D_{s}(S) \cap D_{d}(S)$. We may assume that the sequence $\left\{s_{i}\right\}_{i \in \mathbb{N}}$ is convergent, otherwise we choose a proper subsequence. Let us suppose that $d=s$, then take $b_{i} \in\left(\operatorname{cl} \operatorname{int} D_{S^{\prime}}(S) \backslash \operatorname{int} D_{S^{\prime}}(S)\right) \cap\left[s_{i}, s_{i}+\right.$ $\left.\alpha\left(u+\epsilon_{i}(u-s)-s_{i}\right)\right]$, where $\left.\alpha u+(1-\alpha) s \in \operatorname{int} D_{S^{\prime}}(S), \alpha \in\right] 0,1\left[\right.$, and $s_{i} \notin D_{S^{\prime}}(S)$ and $s_{i} \longrightarrow s$. We have

$$
\begin{array}{r}
\left\|u+\epsilon_{i}(u-s)-s_{i}\right\|<d_{S^{\prime}}\left(u+\epsilon_{i}(u-s)\right) \leq d_{S^{\prime}}\left(b_{i}\right)+\left\|u+\epsilon_{i}(u-s)-b_{i}\right\| \\
=d_{S}\left(b_{i}\right)+\left\|u+\epsilon_{i}(u-s)-b_{i}\right\| \leq\left\|b_{i}-s_{i}\right\|+\left\|u+\epsilon_{i}(u-s)-b_{i}\right\| \\
=\left\|u+\epsilon_{i}(u-s)-s_{i}\right\|
\end{array}
$$

for every $i \in \mathbb{N}$, which is impossible, thus $d \neq s$.

A simple consequence of the Corollary above is

Corollary 5.2 Let $\mathbb{H}$ be a real Hilbert space, $S \subset \mathbb{H}$ be a closed nonempty subset and $\bar{s} \in S$ be such that

$$
\sup _{s \in S, u \in D_{\bar{s}}(S)}\langle s-\bar{s}, u-\bar{s}\rangle>0
$$

and int $D_{\bar{s}}(S) \neq \emptyset$. Then there exists $d \in S, d \neq \bar{s}$ such that $D_{\bar{s}}(S) \cap D_{d}(S) \neq \emptyset$.

Proof Take any $z \in D_{\bar{s}}(S)$ and $s \in S$ such that $\langle s-\bar{s}, z-\bar{s}\rangle>0$ and $] \bar{s}, z\left[\subset\right.$ int $D_{\bar{s}}(S)$. Observe that $\bar{s}+\bar{t}(z-\bar{s}) \in$ fr $D_{\bar{s}}(S)$ for some $\bar{t}>0$ and $\bar{s}+t(z-\bar{s}) \notin$ fr $D_{\bar{s}}(S)$ for every $t>\bar{t}$. Otherwise $\bar{s}+$ cone $(z-\bar{s})=\bar{s}+\left[0, \infty\left[(z-\bar{s}) \subset D_{\bar{s}}(S)\right.\right.$, and consequently

$$
\bigcup_{t>0} B_{\mathbb{H}}[\bar{s}+t(z-\bar{s}), t\|z-\bar{s}\|] \cap S=\{\bar{s}\},
$$

which is impossible, since $\langle s-\bar{s}, z-\bar{s}\rangle>0$. It follows from Proposition 4.5 that there exist $u \in \operatorname{fr} D_{\bar{s}}(S)$ (close to $\bar{s}+\bar{t}(z-\bar{s})$ ) and $x \in \operatorname{int} D_{\bar{s}}(S)$ such that $d_{\mathbb{H} \backslash \operatorname{int} D_{\bar{s}}(S)}(x)=\|u-x\|$ and $] \bar{s}, u\left[\subset\right.$ int $D_{\bar{s}}(S)$ (otherwise $\bar{s}+\operatorname{cone}(z-\bar{s})=\bar{s}+\left[0, \infty\left[(z-\bar{s}) \subset D_{\bar{s}}(S)\right.\right.$ ), hence $\langle u-\bar{s}, u-x\rangle \neq 0$, so it follows from Corollary 5.1 that $u \in D_{d}(S)$ for some $d \in S, d \neq$ $\bar{s}$.

A direct consequence of Corollary 5.2 is that the existence of nonsmooth points in the boundary of a set $S$ can cause that images of the projection mapping $P_{S}(\cdot)$ are not singletons. 
Corollary 5.3 Let $\mathbb{H}$ be a real Hilbert space, $S \subset \mathbb{H}$ be a closed nonempty subset and $\bar{s} \in S$ be such that there are $\varepsilon>0$ and $v^{*} \in S_{\mathbb{H}}[0,1]$ for which

$$
\sup _{s \in S \cap B_{\mathbb{H}}[\bar{s}, \varepsilon]}\left\langle s-\bar{s}, v^{*}\right\rangle+\varepsilon\|s-\bar{s}\| \leq 0
$$

and

$$
\sup _{s^{\prime} \in S}\left\langle s^{\prime}-\bar{s}, v^{*}\right\rangle>0 .
$$

Then there exists $d \in S, d \neq \bar{s}$ such that $D_{\bar{s}}(S) \cap D_{d}(S) \neq \emptyset$.

Proof It is easy to show that $u_{t}:=\bar{s}+t v^{*} \in D_{\bar{s}}(S)$ for $t>0$ small enough, thus

$$
\sup _{s \in S, u \in D_{\bar{s}}(S)}\langle s-\bar{s}, u-\bar{s}\rangle \geq \sup _{s \in S, u_{t} \in D_{\bar{s}}(S)}\left\langle s-\bar{s}, u_{t}-\bar{s}\right\rangle \geq t \sup _{s \in S}\left\langle s-\bar{s}, v^{*}\right\rangle>0
$$

for $t>0$ small enough. In order to apply Corollary 5.2 let us show that int $D_{\bar{s}}(S) \neq \emptyset$. For this reason fix $\delta \in] 0, \min \left\{1, \frac{\varepsilon}{4}\right\}\left[\right.$ and take $z \in B_{\mathbb{H}}\left[v^{*}, \delta\right]$. Observe that if $s \in S \cap B_{\mathbb{H}}[\bar{s}, \varepsilon]$, then

$$
\begin{aligned}
\|\bar{s}+\delta z-s\|^{2} & =\|\delta z\|^{2}+\|\bar{s}-s\|^{2}+ \\
+2 \delta\langle z, \bar{s}-s\rangle & =\|\delta z\|^{2}+\|\bar{s}-s\|^{2} \\
\left.+2 \delta v^{*}, \bar{s}-s\right\rangle+2 \delta\left\langle z-v^{*}, \bar{s}-s\right\rangle \geq\|\delta z\|^{2}+\|\bar{s}-s\|^{2} & \\
& +2 \delta \varepsilon\|\bar{s}-s\|-2 \delta^{2}\|\bar{s}-s\| \geq\|\bar{s}+\delta z-\bar{s}\|^{2} .
\end{aligned}
$$

We have also

$$
\begin{gathered}
\|\bar{s}+\delta z-s\|^{2}=\|\delta z\|^{2}+\|\bar{s}-s\|^{2}+2 \delta\langle z, \bar{s}-s\rangle=\|\delta z\|^{2}+\|\bar{s}-s\|^{2}+2 \delta\left\langle v^{*}, \bar{s}-s\right\rangle \\
+2 \delta\left\langle z-v^{*}, \bar{s}-s\right\rangle \geq\|\delta z\|^{2}+\|\bar{s}-s\|^{2}-2 \delta\|\bar{s}-s\|-2 \delta^{2}\|\bar{s}-s\| \\
\geq\|\delta z\|^{2}+\|\bar{s}-s\|(\|\bar{s}-s\|-4 \delta) \geq\|\bar{s}+\delta z-\bar{s}\|^{2},
\end{gathered}
$$

whenever $s \in S \cap\left(\mathbb{H} \backslash B_{\mathbb{H}}[\bar{s}, \varepsilon]\right)$, thus $\bar{s}+\delta B_{\mathbb{H}}\left[v^{*}, \delta\right] \subset D_{\bar{s}}(S)$, so it follows from Corollary 5.1 that $u \in D_{d}(S)$ for some $d \in S, d \neq \bar{s}$.

Corollary 5.4 Let $\mathbb{H}$ be a real Hilbert space, $S^{\prime} \subset S \subset \mathbb{H}$ be closed nonempty subsets and $S^{\prime} \in W(S)$, see (4), be such that $\bar{u} \in D_{S^{\prime}}(S),\|\bar{u}-\bar{s}\|=d_{S}(\bar{u})>0, \bar{s} \in S^{\prime},\left(S \backslash S^{\prime}\right) \neq \varnothing$ and

$$
\exists \delta>0: \forall s \in \mathbb{B}_{\mathbb{H}}[\bar{s}, \delta] \cap S^{\prime}, \exists \epsilon>0: \mathbb{B}_{\mathbb{H}}[s, \epsilon] \cap\left(S \backslash S^{\prime}\right)=\emptyset .
$$

If $D_{s_{1}}(S) \cap D_{s_{2}}(S)=\emptyset$ for all $s_{1}, s_{2} \in S^{\prime}, s_{1} \neq s_{2}$, then there exists $d \in S, d \notin S^{\prime}$ such that $D_{S^{\prime}}(S) \cap D_{d}(S) \neq \emptyset$.

Proof It follows from Lemma 4.8 and Proposition 4.5 that there are $u \in \operatorname{fr} D_{S^{\prime}}(S), s \in$ $S^{\prime} \cap B_{\mathbb{H}}[\bar{s}, \delta]$ and $x \in \mathbb{H} \backslash S$ such that $d_{\mathbb{H} \backslash \operatorname{int} D_{S^{\prime}}(S)}(x)=\|u-x\|, d_{S^{\prime}}(u)=\|u-s\|>0$. We recall that $z \in \mathbb{H} \backslash D_{S^{\prime}}(S)$ if and only if $d_{S^{\prime}}(z)>d_{S}(z)$, since $S^{\prime} \subset S$. Take sequences $\left\{u_{i}\right\}_{i \in \mathbb{N}}$ in $\mathbb{H} \backslash D_{S^{\prime}}(S), u_{i} \longrightarrow u$, and $\left\{s_{i}\right\}_{i \in \mathbb{N}}$ in $S$ such that $\left\|u_{i}-s_{i}\right\| \longrightarrow d_{S}(u)$ and

$$
d_{S^{\prime}}\left(u_{i}\right)>\left\|u_{i}-s_{i}\right\|
$$

for every $i \in \mathbb{N}$. It follows from (21) that $s_{i} \in S \backslash S^{\prime}$ for every $i \in \mathbb{N}$. It is easy to observe that weakly closed subsets are approximately compact, thus using Lemma 2.8 and Proposition 3.1 we infer that the sequence $\left\{s_{i}\right\}_{i \in \mathbb{N}}$ has a convergent subsequence, say $s_{i_{k}} \longrightarrow d$. If $d=s$, then using (20) we obtain $s_{i_{k}} \in S^{\prime}$ for $k \in \mathbb{N}$ large enough, but this is impossible in view of (21). Of course $u \in D_{d}(S)$, and consequently $u \in D_{d}(S) \cap D_{S^{\prime}}(S)$. 
Corollary 5.5 Let $\mathbb{H}$ be a real Hilbert space, $S \subset \mathbb{H}$ be a closed nonempty subset, and $S^{\prime} \subset S$ be a closed convex nonempty subset such that int $D_{S^{\prime}}(S) \backslash S^{\prime} \neq \emptyset$ and cl int $D_{S^{\prime}}(S) \backslash$ $\left(\right.$ int $\left.D_{S^{\prime}}(S) \cup S^{\prime}\right) \neq \emptyset$. Then

$$
\left.(\mathbb{H} \backslash S) \cap\left(\operatorname{clint} D_{S^{\prime}}(S) \backslash\left(\operatorname{int} D_{S^{\prime}}(S) \cup S^{\prime}\right)\right)\right) \subset \operatorname{cl}\left(\bigcup_{d \in \operatorname{cl}\left(S \backslash S^{\prime}\right)} D_{d}(S) \cap D_{S^{\prime}}(S)\right) \backslash S .
$$

Proof It follows from Proposition 4.5 that for every $w \in \operatorname{cl}$ int $D_{S^{\prime}}(S) \backslash$ int $D_{S^{\prime}}(S)$ and every $\mu>0$ there is $u \in\left(\operatorname{clint} D_{S^{\prime}}(S) \backslash \operatorname{int} D_{S^{\prime}}(S)\right) \cap \mathbb{B}_{\mathbb{H}}[w, \mu]$ such that $d_{\mathbb{H} \backslash \operatorname{int} D_{S^{\prime}}(S)}(x)=$ $\|u-x\|$ for some $x \in \operatorname{int} D_{S^{\prime}}(S)$. Fix some $u^{\prime} \in \operatorname{cl}$ int $D_{S^{\prime}}(S) \backslash\left(\right.$ int $D_{S^{\prime}}(S) \cup S^{\prime}$ ) such that $d_{\mathbb{H} \backslash \text { int }} D_{S^{\prime}}(S)\left(x^{\prime}\right)=\left\|u^{\prime}-x^{\prime}\right\|$ for some $x^{\prime} \in \operatorname{int} D_{S^{\prime}}(S)$. If there is $t^{\prime}>0$ such that $u^{\prime}+t^{\prime}\left(u^{\prime}-P_{S^{\prime}}\left(u^{\prime}\right)\right) \in D_{P_{S^{\prime}}\left(u^{\prime}\right)}(S)$, then $u^{\prime}+t^{\prime}\left(u^{\prime}-P_{S^{\prime}}\left(u^{\prime}\right)\right) \notin$ int $D_{P_{S^{\prime}}\left(u^{\prime}\right)}(S)$, otherwise $u^{\prime} \in \operatorname{int} D_{P_{S^{\prime}}\left(u^{\prime}\right)}(S)$ by the convexity of $D_{S^{\prime}}(S)$ which contradicts the choice of $u^{\prime} \in \mathrm{cl}$ int $D_{S^{\prime}}(S) \backslash\left(\right.$ int $\left.D_{S^{\prime}}(S) \cup S^{\prime}\right)$. Thus there is a sequence $\left\{u_{i}^{\prime}\right\}_{i \in \mathbb{N}}$ in $\mathbb{H} \backslash D_{S^{\prime}}(S)$ such that $u_{i}^{\prime} \longrightarrow u$. There are $s_{i}^{\prime} \in S \backslash S^{\prime}$ such that $\left\|u^{\prime}-s_{i}^{\prime}\right\| \longrightarrow d_{S}\left(u^{\prime}\right)$. It follows from Lemma 2.4 that the sequence $\left\{s_{i}^{\prime}\right\}_{i \in \mathbb{N}}$ is convergent, say to $d \in \operatorname{cl}\left(S \backslash S^{\prime}\right)$, so we are done. Now let us consider the case $u^{\prime}+t^{\prime}\left(u^{\prime}-P_{S^{\prime}}\left(u^{\prime}\right)\right) \notin D_{P_{S^{\prime}}\left(u^{\prime}\right)}(S)$ for every $t^{\prime}>0$ and apply Corollary 5.1 to get the statement. For this reason let us observe that if $P_{S^{\prime}}\left(u^{\prime}\right) \in \operatorname{cl}\left(S \backslash S^{\prime}\right)$, then we are done, however if $P_{S^{\prime}}\left(u^{\prime}\right) \notin \operatorname{cl}\left(S \backslash S^{\prime}\right)$ then $\left\langle u^{\prime}-x^{\prime}, u^{\prime}-P_{S^{\prime}}\left(u^{\prime}\right)\right\rangle>0$, and all assumptions of Corollary 5.1 are fulfilled, so the statement is a consequence of Corollary 5.1.

\section{The Vlasov Condition}

In this section we present some results related to the Vlasov condition, see (6.1) below. In order to characterize the role of the Vlasov condition let us start with the following result which was obtained by L. P. Vlasov [34, Theorem 3] in the setting of a Banach space $(X,\|\cdot\|)$ whose dual norm $\|\cdot\|_{*}$ of $X^{*}$ was assumed to be strictly convex. It states that in some Banach spaces the continuity of the metric projection on a set $S$ preserves the convexity of the set. A new proof of this result for $X$ being a Banach space whose norm is uniformly Gâteaux differentiable is given in [23, Theorem 4.4]. In the next section the Vlasov condition is applied to get convexity of Chebyshev sets in some new cases.

Theorem 6.1 Let $X$ be a Banach space whose norm is uniformly Gâteaux differentiable and $S \subset X$ be a Chebyshev set with continuous metric projection. Then $S$ is convex.

The continuity of the metric projection can be also preserved by checking if the Vlasov condition is satisfied, see [35, page 56] and also [33, 34], i.e.

$$
\limsup _{y \rightarrow 0} \frac{d_{S}(x+y)-d_{S}(x)}{\|y\|}=1
$$

for all $x \notin S$.

Corollary 6.2 Let $\mathbb{H}$ be a real Hilbert space, $S \subset \mathbb{H}$ be a nonempty subset. Assume that $x \notin \operatorname{cl} S$ is such that there are $\left\{w_{1}, w_{2}, \ldots\right\} \subset \operatorname{cl} S, w_{i} \stackrel{\text { weak }}{\longrightarrow} s^{*} \in \mathbb{H}$, and $x_{i} \in D_{w_{i}}(S)$ such that $\bigcup_{t \in] 0, \infty[} B\left[w_{i}+t\left(x_{i}-w_{i}\right), t\left\|x_{i}-w_{i}\right\|\right] \cap S=\left\{w_{i}\right\}$ for every $i \in \mathbb{N}$, and $x=\lim _{i \rightarrow \infty} x_{i}$. Then the Vlasov condition is satisfied at $x$. 


\section{Proof Since}

for every $i \in \mathbb{N}$, so

$$
\bigcup_{t \in] 0, \infty[} B\left[w_{i}+t\left(x_{i}-w_{i}\right), t\left\|x_{i}-w_{i}\right\|\right] \cap S=\left\{w_{i}\right\}
$$

$$
\forall i \in N, \forall s \in S,\left\langle x_{i}-w_{i}, s-w_{i}\right\rangle \leq 0
$$

Hence

$$
\begin{array}{r}
\left\|x-s^{*}\right\|^{2} \geq \liminf _{i \longrightarrow \infty}\left\|x_{i}-s^{*}\right\|^{2}=\liminf _{i \longrightarrow \infty}\left\|x_{i}-w_{i}\right\|^{2}+2\left\langle x_{i}-w_{i}, w_{i}-s^{*}\right\rangle+\left\|w_{i}-s^{*}\right\|^{2} \\
\geq \liminf _{i \longrightarrow \infty} d_{S}\left(x_{i}\right)^{2}+\left\|w_{i}-s^{*}\right\|^{2} \geq d_{S}^{2}(x)>0,
\end{array}
$$

so $x \neq s^{*}$. For every $t>0$ there is $s_{t} \in S$ such that $d_{S}^{2}\left(x+t\left(x-s^{*}\right)\right)+t^{2} \geq \| x+t(x-$ $\left.s^{*}\right)-s_{t} \|^{2}$, so

$$
\begin{gathered}
t^{2}+d_{S}^{2}\left(x+t\left(x-s^{*}\right)\right) \geq\left\|x+t\left(x-s^{*}\right)-s_{t}\right\|^{2}=\left\|x+t\left(x-s^{*}\right)-s^{*}+s^{*}-s_{t}\right\|^{2} \\
=\left\|x+t\left(x-s^{*}\right)-s^{*}\right\|^{2}+2\left\langle x+t\left(x-s^{*}\right)-s^{*}, s^{*}-s_{t}\right\rangle+\left\|s^{*}-s_{t}\right\|^{2} \\
=(1+t)^{2}\left\|x-s^{*}\right\|^{2}+(1+t) 2\left\langle x-s^{*}, s^{*}-s_{t}\right\rangle+\left\|s^{*}-s_{t}\right\|^{2} \\
\left.=(1+t)^{2}\left(\left\|x-s^{*}\right\|^{2}+2\left\langle x-s^{*}, s^{*}-s_{t}\right\rangle\right)+\left\|s^{*}-s_{t}\right\|^{2}\right) \\
-2 t\left(\left\langle x-s^{*}, s^{*}-s_{t}\right\rangle+\left\|s^{*}-s_{t}\right\|^{2}\right)-t^{2}\left(2\left\langle x-s^{*}, s^{*}-s_{t}\right\rangle+\left\|s^{*}-s_{t}\right\|^{2}\right),
\end{gathered}
$$

we recall that $x=\lim _{i \rightarrow \infty} x_{i}, d_{S}(x)=\lim _{i \rightarrow \infty} d_{S}\left(x_{i}\right)=\lim _{i \rightarrow \infty}\left\|x_{i}-w_{i}\right\|$, which implies the inequality

whenever

$$
\lim _{t \downarrow 0} \frac{d_{S}^{2}\left(x+t\left(x-s^{*}\right)\right)-d_{S}^{2}(x)}{t\left\|x-s^{*}\right\|} \geq 2 d_{S}(x),
$$

$$
\liminf _{t \downarrow 0}\left\langle x-s^{*}, s^{*}-s_{t}\right\rangle+\left\|s^{*}-s_{t}\right\|^{2} \leq 0,
$$

since $\left\|x-s^{*}\right\| \leq d_{S}(x)$. Observe that (24) implies (22), so it is enough to get inequality (25) in order to finish the proof. Suppose the contrary, that is

$$
\liminf _{t \downarrow 0}\left\langle x-s^{*}, s^{*}-s_{t}\right\rangle+\left\|s^{*}-s_{t}\right\|^{2} \geq 2 \epsilon,
$$

for some $\epsilon>0$. By (23) and (26) we have

$$
\left\langle x_{i}-w_{i}, s_{t}-w_{i}\right\rangle \leq 0 \text { and }\left\langle x-s_{t}, s^{*}-s_{t}\right\rangle \geq \frac{3}{2} \epsilon
$$

for $t>0$ small enough, so

$$
\left\|x_{i}-w_{i}\right\|^{2}+\left\langle x_{i}-w_{i}, s_{t}-x_{i}\right\rangle \leq 0 \text { and }\left\|x-s_{t}\right\|^{2}+\left\langle x-s_{t}, s^{*}-x\right\rangle \geq \frac{3}{2} \epsilon,
$$

hence

$$
-\left\|x_{i}-w_{i}\right\|^{2}-\left\langle x_{i}-w_{i}, s_{t}-x\right\rangle+\left\|x-s_{t}\right\|^{2}+\left\langle x-s_{t}, s^{*}-x\right\rangle \geq \epsilon,
$$

which implies

$$
\begin{array}{r}
\epsilon \leq \liminf _{t \downarrow 0} \liminf _{i \rightarrow \infty}\left(\left\langle x-s_{t}, s^{*}-w_{i}\right\rangle+\left(\left\|x-s_{t}\right\|^{2}-\left\|x_{i}-w_{i}\right\|^{2}\right)+\left\langle x-s_{t}, x_{i}-x\right\rangle\right) \\
=\liminf _{t \downarrow 0}\left(\left\|x-s_{t}\right\|^{2}-d_{S}^{2}(x)\right)=0,
\end{array}
$$

a contradiction. 


\section{Tangent Cones to Preimages of the Metric Projection on a Set of Isolated Points}

Let $S \subset \mathbb{H}$ be a subset of isolated points, $u \notin S$ and $\{\bar{s}\}=P_{S}(u)$. In this section the tangent cone to $P_{S}^{-1}(\bar{s})$ is calculated, whenever $\bar{s}$ is not a weak cluster point of $S$. Let us recall that for every nonempty subset $A \subset \mathbb{H}$ and $a \in \operatorname{cl} A$ by

$$
T(A, a):=\left\{z \in \mathbb{H} \mid \forall i \in \mathbb{N}, a+t_{i} z_{i} \in A, \text { where } t_{i} \downarrow 0, z_{i} \longrightarrow z\right\}
$$

we denote the tangent cone (Bouligand tangent cone or the contingent cone, see [6, Exercise 5.2.21, page 209]).

Theorem 7.1 Let $\mathbb{H}$ be a real Hilbert space, $u \in \mathbb{H}$ be given, $S=\left\{\bar{s}, s_{1}, s_{2}, \ldots\right\} \subset \mathbb{H}$ be a closed subset of isolated points (this implies that $S$ is a closed subset) such that:

1. $\left\|u-s_{i}\right\| \longrightarrow\|u-\bar{s}\|$;

2. $\forall i \in \mathbb{H},\left\|u-s_{i}\right\|>\left\|u-s_{i+1}\right\|>\|u-\bar{s}\|$;

3. $s_{i} \stackrel{\text { weak }}{\longrightarrow} s^{*} \in B_{\mathbb{H}}[u,\|u-\bar{s}\|]$

then $T\left(D_{\bar{s}}\left(\left\{\bar{s}, s_{1}, s_{2}, \ldots\right\}\right), u\right)=\left\{z \in \mathbb{H}:\left\langle z, s^{*}-\bar{s}\right\rangle \leq 0\right\}$.

Proof Observe that it follows from assumptions 1. and 3. that $s^{*}-\bar{s}=\alpha(u-\bar{s})+w$, where $\alpha>0$ and $\langle w, u-\bar{s}\rangle=0$. Let us also notice that

$$
\text { int } D_{\bar{s}}(S) \neq \emptyset \text { and } u \in \text { fr } D_{\bar{s}}(S) \text {. }
$$

Assumption 2. gives $u \in D_{\bar{s}}(S) \backslash$ int $D_{\bar{s}}(S)$. Fix any $z \in T\left(\right.$ fr $\left.D_{\bar{s}}(S), u\right)$ and take sequences $\left\{u_{i}\right\}_{i \in \mathbb{N}}$ in fr $D_{\bar{s}}(S)$ and $\left\{x_{i}\right\}_{i \in \mathbb{N}}$ in int $D_{\bar{s}}(S)$ such that

$$
\begin{aligned}
& \forall i \in \mathbb{N},\left\|u_{i}-x_{i}\right\|=d_{\mathbb{H} \backslash \operatorname{int} D_{\bar{s}}(S)}\left(x_{i}\right) \text { and } z=\lim _{i \rightarrow \infty}\left\|u_{i}-u\right\|^{-1}\left(u_{i}-u\right), \\
& \qquad u=\lim _{i \longrightarrow \infty} u_{i} .
\end{aligned}
$$

The choice of the sequences is preserved by Proposition 4.5 and Lemma 2.8. It follows from Proposition 3.1 that either there is a converging subsequence of the sequence $\left\{s_{i}\right\}_{i \in \mathbb{N}}$ (but this is impossible) or for each $i$ there is $k(i) \in \mathbb{N}$ such that (keep in mind that $u_{i} \in \operatorname{fr} D_{\bar{s}}(S)$ is a "smooth" point)

$$
\left\|u_{i}-s_{k(i)}\right\|=\left\|u_{i}-\bar{s}\right\| .
$$

Because of assumption 2, there is a subsequence $\left\{i_{n}\right\}_{n \in \mathbb{N}}$ such that $k\left(i_{n}\right)<k\left(i_{n+1}\right)$. Put $y_{n}:=u_{i_{n}}$ and $d_{n}:=s_{k\left(i_{n}\right)}$. For every $n \in \mathbb{N}$ we have

$$
\left\|y_{n}-\bar{s}\right\|^{2}=\left\|y_{n}-d_{n}\right\|^{2}
$$

and

$$
\begin{array}{r}
\left\|y_{n}-d_{n}\right\|^{2}=\left\|y_{n}-u+u-d_{n}\right\|^{2}=\left\|y_{n}-u\right\|^{2}+2\left\langle y_{n}-u, u-d_{n}\right\rangle+\left\|u-d_{n}\right\|^{2} \\
\geq\left\|y_{n}-u\right\|^{2}+2\left\langle y_{n}-u, u-d_{n}\right\rangle+\|u-\bar{s}\|^{2}=\left\|y_{n}-u\right\|^{2}+2\left\langle y_{n}-u, u-d_{n}\right\rangle \\
+\left\|u-y_{n}\right\|^{2}+2\left\langle u-y_{n}, y_{n}-\bar{s}\right\rangle+\left\|y_{n}-\bar{s}\right\|^{2},
\end{array}
$$


thus by (28) we get

$$
0 \geq\left\|u-y_{n}\right\|+\left\langle\left\|u-y_{n}\right\|^{-1}\left(y_{n}-u\right), u-d_{n}-y_{n}+\bar{s}\right\rangle .
$$

Passing to the limit by assumption 3 . we get

$$
0 \geq\left\langle z, \bar{s}-s^{*}\right\rangle \text {. }
$$

In the next step we show that $T\left(\operatorname{fr} D_{\bar{s}}(S), u\right)=\left\{z \in \mathbb{H}:\left\langle z, s^{*}-\bar{s}\right\rangle=0\right\}$. First let us establish the inclusion $T$ (fr $\left.D_{\bar{s}}(S), u\right) \subset\left\{z \in \mathbb{H}:\left\langle z, s^{*}-\bar{s}\right\rangle=0\right\}$. For this reason let us suppose the contrary, i.e. there is $\bar{z} \in T\left(\operatorname{fr} D_{\bar{s}}(S), u\right)$ such that $\left\langle\bar{z}, s^{*}-\bar{s}\right\rangle>0$ (keep in mind (29)). Of course we have

$$
\lim _{t \downarrow 0} t^{-1} d_{D_{\bar{s}}(S)}(u+t \bar{z})=0 .
$$

If $u-\bar{t} \bar{z} \in$ int $D_{\bar{s}}(S)$ for some $\bar{t}>0$, then there are $\bar{\mu}>0, \alpha_{i}>0, z_{i} \in \mathbb{H}$ such that $\alpha_{i} \downarrow 0$, $z_{i} \longrightarrow \bar{z}$, and

$$
u-\bar{t} \mathbb{B}_{\mathbb{H}}[\bar{z}, \bar{\mu}] \subset \text { int } D_{\bar{s}}(S), u+\alpha_{i} z_{i} \in D_{\bar{s}}(S) .
$$

It follows from the convexity of $D_{\bar{s}}(S)$ that

$$
u \in u+\frac{\alpha_{i} \bar{t}}{\alpha_{i}+\bar{t}}\left(z_{i}-\bar{z}+\mathbb{B}_{\mathbb{H}}[0, \bar{\mu}]\right) \subset \operatorname{int} D_{\bar{s}}(S),
$$

but this is impossible since (27). Thus there are sequences $\left\{t_{i}\right\}_{i \in \mathbb{N}}$ in $\left.] 0, \infty\right],\left\{\delta_{i}\right\}_{i \in \mathbb{N}}$ in $[0, \infty]$ such that that $t_{i} \downarrow 0$ and $\lim _{i \rightarrow \infty} \delta_{i}=\delta$ or $\beta:=\lim _{i \rightarrow \infty} t_{i} \delta_{i} \leq 1$, and

$$
\forall i \in \mathbb{N}, u-t_{i}\left(\bar{z}-\delta_{i}(\bar{s}-u)\right) \in \text { fr } D_{\bar{s}}(S) .
$$

So if $\delta<\infty$, then $-\bar{z}+\delta(\bar{s}-u) \in T\left(\operatorname{fr} D_{\bar{s}}(S), u\right)$ and using (29) with $-\bar{z}+\delta(\bar{s}-u)$ instead of $z$ we get

$$
\begin{aligned}
0 \leq\left\langle s^{*}-\bar{s},-\bar{z}+\delta(\bar{s}-u)\right\rangle=\left\langle s^{*}-\bar{s},-\bar{z}\right\rangle+\langle\alpha(u-\bar{s})+w, \delta(\bar{s}-u)\rangle \\
=\left\langle s^{*}-\bar{s},-\bar{z}\right\rangle+\langle\alpha(u-\bar{s}), \delta(\bar{s}-u)\rangle=\left\langle s^{*}-\bar{s},-\bar{z}\right\rangle-\alpha \delta\|u-\bar{s}\|^{2}<0,
\end{aligned}
$$

a contradiction, thus the inclusion $T$ (fr $\left.D_{\bar{s}}(S), u\right) \subset\left\{z \in \mathbb{H}:\left\langle z, s^{*}-\bar{s}\right\rangle=0\right\}$ holds true. If $\delta=\infty$, then by (30) $(1-\beta) u+\beta \bar{s} \in$ fr $D_{\bar{s}}(S)$, which is possible only for $\beta=0$. Hence, by (30) we get $\bar{s}-u \in T$ (fr $\left.D_{\bar{s}}(S), u\right)$, but this is impossible.

In order to establish the reverse inclusion $\left\{z \in \mathbb{H}:\left\langle z, s^{*}-\bar{s}\right\rangle=0\right\} \subset T\left(\right.$ fr $\left.D_{\bar{s}}(S), u\right)$, let us fix any $h \in\left\{z \in \mathbb{H}:\left\langle z, s^{*}-\bar{s}\right\rangle=0\right\}$. If $h \notin T\left(D_{\bar{s}}(S), u\right)$ then there are sequences $\left\{\gamma_{i}\right\}_{i \in \mathbb{N}}$ in $] 0, \infty]$ and $\left\{\theta_{i}\right\}_{i \in \mathbb{N}}$ in $[0, \infty]$ such that that $\lim _{i \rightarrow \infty} \theta_{i}=\theta$ or $\zeta:=\lim _{i \rightarrow \infty} \gamma_{i} \theta_{i} \leq 1$, and

$$
\forall i \in \mathbb{N}, u+\gamma_{i}\left(h+\theta_{i}(\bar{s}-u)\right) \in \operatorname{fr} D_{\bar{s}}(S) .
$$

So if $\theta<\infty$ then $h+\theta(\bar{s}-u) \in T\left(\right.$ fr $\left.D_{\bar{s}}(S), u\right)$ and

$$
\begin{aligned}
0=\left\langle s^{*}-\bar{s}, h\right. & +\theta(\bar{s}-u)\rangle=\left\langle s^{*}-\bar{s}, h\right\rangle+\langle\alpha(u-\bar{s})+w, \theta(\bar{s}-u) \\
& =\left\langle s^{*}-\bar{s}, h\right\rangle+\left\langle\alpha(u-\bar{s}), \theta(\bar{s}-u)=\left\langle s^{*}-\bar{s}, h\right\rangle-\alpha \theta\|u-\bar{s}\|^{2}<0,\right.
\end{aligned}
$$

a contradiction, thus $h \in T\left(D_{\bar{s}}(S), u\right)$. If $\theta=\infty$ then by (31) we get $(1-\zeta) u+$ $\zeta \bar{s} \in$ fr $D_{\bar{s}}(S)$, which implies $\zeta=0$, since $\bar{s} \in$ int $D_{\bar{s}}(S)$. Again using (31) we get $\bar{s}-u \in T\left(\right.$ fr $\left.D_{\bar{s}}(S), u\right)$, which is impossible. Similarly we have $-h \in T\left(D_{\bar{s}}(S), u\right)$. If $h \in \operatorname{int} T\left(D_{\bar{s}}(S), u\right)$ (or $-h \in \operatorname{int} T\left(D_{\bar{s}}(S), u\right)$ ) then there is $t_{w}>0$ such that $u+t_{w} h \in$ int $D_{\bar{s}}(S)$ (or $u-t_{w} h \in$ int $D_{\bar{s}}(S)$, respectively), then $u \in$ int $D_{\bar{s}}(S)$, by the convexity of $D_{\bar{s}}(S)$, since $-h \in T\left(D_{\bar{s}}(S), u\right)$ (or $h \in T\left(D_{\bar{S}}(S), u\right)$, respectively) but this is impossible since (27). Thus $h \in \operatorname{fr} T\left(D_{\bar{s}}(S), u\right)=T\left(\operatorname{fr} D_{\bar{S}}(S), u\right)$, which establishes the equality

$$
\left\{z \in \mathbb{H}:\left\langle z, s^{*}-\bar{s}\right\rangle=0\right\}=T\left(\text { fr } D_{\bar{s}}(S), u\right) .
$$


In order to finish the proof let us observe that for all $t>0, z \in T\left(D_{\bar{s}}(S), u\right)$ and $i \in \mathbb{N}$ we have

$$
\begin{aligned}
d_{\bar{s}}^{2}(u+t z) \leq\left(\left\|u+t z-s_{i}\right\|\right. & \left.+2 d_{D_{\bar{s}}(S)}(u+t z)\right)^{2}=\left\|u-s_{i}\right\|^{2}+2 t\left\langle z, u-s_{i}\right\rangle+t^{2}\|z\|^{2} \\
& +2 d_{D_{\bar{s}}(S)}(u+t \bar{z})\left(2 d_{D_{\bar{s}}(S)}(u+t \bar{z})+2\left\|u+t z-s_{i}\right\|\right) .
\end{aligned}
$$

Thus assumptions from 1. to 3 . ensure that

$$
\begin{aligned}
d_{\bar{s}}^{2}(u+t z) \leq d_{\bar{s}}^{2}(u)+2 t\left\langle z, u-s^{*}\right\rangle+t^{2}\|z\|^{2} & \\
+ & 2 d_{D_{\bar{s}}(S)}(u+t \bar{z})\left(2 d_{D_{\bar{s}}(S)}(u+t \bar{z})+2 \sup _{i \in \mathbb{N}}\left\|u+t z-s_{i}\right\|\right),
\end{aligned}
$$

consequently

$$
\langle z, u-\bar{s}\rangle \leq\left\langle z, u-s^{*}\right\rangle
$$

for every $z \in T\left(D_{\bar{S}}(S), u\right)$, so we have the inclusion

$$
T\left(D_{\bar{s}}(S), u\right) \subset\left\{z \in \mathbb{H}:\left\langle z, s^{*}-\bar{s}\right\rangle \leq 0\right\} .
$$

Observe that if $\left\langle z, s^{*}-\bar{s}\right\rangle<0$, then, by assumption 3., $\left\langle\bar{s}-u, \bar{s}-s^{*}\right\rangle>0$, so using (32) we get

$$
z+\mu(u-\bar{s}) \in \operatorname{fr} T\left(D_{\bar{s}}(S), u\right)=T\left(\operatorname{fr} D_{\bar{s}}(S), u\right)
$$

for some $\mu>0$. Since $\mu(\bar{s}-u) \in T\left(D_{\bar{S}}(S), u\right)$, the convexity of $T\left(D_{\bar{S}}(S), u\right)$ gives $z \in T\left(D_{\bar{s}}(S), u\right)$. Hence

$$
\left\{z \in \mathbb{H}:\left\langle z, s^{*}-\bar{s}\right\rangle \leq 0\right\} \subset T\left(D_{\bar{s}}(S), u\right)
$$

and the proof is finished.

\section{Some Sufficient Conditions for the Convexity of Chebyshev Sets}

The problem of convexity of Chebyshev sets of a Hilbert space is old, we refer to [26, K4 Farthest and Nearest Points in Hilbert Space, Comments by Grünbaum] for some historical aspects. In this section we exhibit some kinds of subsets in Hilbert space which are convex whenever they are Chebyshev. First let us recall that due to V. Klee we know that weakly closed Chebyshev sets are convex, see [24, 25]. Another way to get the convexity of a Chebyshev set is to assume a differentiability of the distance function outside the set. Namely, if the distance function to a Chebyshev set is Fréchet differentiable at all points outside the set then the set is convex too, we refer to [13, 14, 18, 19] for details. Due to L. P. Vlasov we know also that the continuity of the metric projection (which implies the convexity) can be obtained by checking if the Vlasov condition is satisfied, see (22) and [35, page $56]$, we refer also to $[33,34]$. The Vlasov result is also important in the proof of Theorem 8.10. At this moment it seems that this tool is essential in detecting whether a Chebyshev set is convex or not. In order to shed light on the meaning of the Vlasov condition let us observe that if it is violated in some special points then the set cannot be a Chebyshev set, namely

Lemma 8.1 Let $\mathbb{H}$ be a real Hilbert space with, $S \subset \mathbb{H}$ be a nonempty subset. Assume that $u \in D_{s}(S), u \neq s$ is such that

$$
\gamma:=\lim _{t \downarrow 0} \frac{d_{S}(u+t(u-s))-d_{S}(u)}{t\|u-s\|} \leq 1 .
$$


Then for all sequences $\left\{s_{i}\right\}_{i \in \mathbb{N}}$ in $S,\left\{\epsilon_{i}\right\}_{i \in \mathbb{N}}$ in $] 0, \infty\left[\right.$ such that $\lim _{i \rightarrow \infty} \epsilon_{i}=0$ and

$$
\left\|u+\epsilon_{i}\right\| u-s\left\|^{-1}(u-s)-s_{i}\right\|<\sqrt{d_{S}^{2}\left(u+\epsilon_{i}\|u-s\|^{-1}(u-s)\right)+\epsilon_{i}^{2}} .
$$

we have

$$
\liminf _{i \rightarrow \infty}\left\|s_{i}-s\right\| \geq \sqrt{2(1-\gamma)} d_{S}(u) .
$$

Proof If

$$
\lim _{t \downarrow 0} \frac{d_{S}(u+t(u-s))-d_{S}(u)}{t\|u-s\|}=1
$$

then the statement is obvious. Let us suppose that $s \in S, u \in D_{s}(S)$, and

$$
\lim _{t \downarrow 0} \frac{d_{S}(u+t(u-s))-d_{S}(u)}{t\|u-s\|}<1 .
$$

Take sequences $\left\{\epsilon_{i}\right\}_{i \in \mathbb{N}}$ in $] 0, \infty\left[,\left\{s_{i}\right\}_{i \in \mathbb{N}}\right.$ in $S,\left\{\alpha_{i}\right\}_{i \in \mathbb{N}}$ in $\left[0, \infty\left[,\left\{w_{i}\right\}_{i \in \mathbb{N}}\right.\right.$ in $\mathbb{H}$ such that $\lim _{i \rightarrow \infty} \epsilon_{i}=0, s_{i}-s=\alpha_{i}(u-s)+w_{i},\left\langle w_{i}, u-s\right\rangle=0$, and

$$
\begin{aligned}
& d_{S}^{2}\left(u+\epsilon_{i}\|u-s\|^{-1}(u-s)\right) \leq r^{2}+2 r \gamma \epsilon_{i}+\epsilon_{i}^{2} \\
& \quad \text { and }\left\|u+\epsilon_{i}\right\| u-s\left\|^{-1}(u-s)-s_{i}\right\|^{2}<d_{S}^{2}\left(u+\epsilon_{i}\|u-s\|^{-1}(u-s)\right)+\epsilon_{i}^{2}
\end{aligned}
$$

for every $i \in \mathbb{N}$ (we can assume this without loss of generality, otherwise we omit a finite number of indices), where $r:=\|u-s\|$ and of course $u+\epsilon_{i}\|u-s\|^{-1}(u-s) \notin D_{s}(S)$, otherwise $\lim _{t \downarrow 0} \frac{d_{S}(u+t(u-s))-d_{S}(u)}{t\|u-s\|}=1$. We have

$$
\begin{aligned}
\gamma=\lim _{t \downarrow 0} \frac{d_{S}(u+t(u-s))-}{d_{S}(u)} & \\
t\|u-s\| & \leq \lim _{i \rightarrow \infty} \frac{\left\|u+\epsilon_{i}\right\| u-s\left\|^{-1}(u-s)-s_{i}\right\|-d_{S}(u)}{\epsilon_{i}} \leq \gamma,
\end{aligned}
$$

as well as

$$
\begin{array}{r}
\left\|u-s_{i}\right\|^{2} \geq r^{2} \Longrightarrow\|u-s\|^{2}+2\left\langle u-s, s-s_{i}\right\rangle+\left\|s-s_{i}\right\|^{2} \geq r^{2} \\
\Longrightarrow\left\|s-s_{i}\right\|^{2} \geq 2\left\langle u-s, s_{i}-s\right\rangle \Longrightarrow\left\|w_{i}\right\|^{2}+\alpha_{i}^{2} r^{2} \geq 2\left\langle u-s, s_{i}-s\right\rangle \\
\Longrightarrow\left\|w_{i}\right\|^{2} \geq r^{2}\left(2 \alpha_{i}-\alpha_{i}^{2}\right)
\end{array}
$$

and by the last inequality in (34) we obtain

$$
\begin{gathered}
2 \epsilon_{i}^{2}+r^{2}+2 r \gamma \epsilon_{i} \geq \epsilon_{i}^{2}+d_{S}^{2}\left(u+\epsilon_{i}\|u-s\|^{-1}(u-s)\right)>\left\|u+\epsilon_{i}\right\| u-s\left\|^{-1}(u-s)-s_{i}\right\|^{2} \\
=\left\|u+\epsilon_{i}\right\| u-s\left\|^{-1}(u-s)-s+s-s_{i}\right\|^{2}=\left\|u+\epsilon_{i}\right\| u-s\left\|^{-1}(u-s)-s\right\|^{2} \\
+2\left\langle u+\epsilon_{i}\|u-s\|^{-1}(u-s)-s, s-s_{i}\right\rangle+\left\|s-s_{i}\right\|^{2}=\left(r+\epsilon_{i}\right)^{2}-2 r \alpha_{i}\left(r+\epsilon_{i}\right) \\
+\alpha_{i}^{2} r^{2}+\left\|w_{i}\right\|^{2} \geq\left(r+\epsilon_{i}\right)^{2}-2 r \alpha_{i}\left(r+\epsilon_{i}\right)+\alpha_{i}^{2} r^{2}+r^{2}\left(2 \alpha_{i}-\alpha_{i}^{2}\right)
\end{gathered}
$$

thus

$$
\gamma+\alpha_{i} \geq 1-\frac{\epsilon_{i}}{2 r}
$$

so using (34) we get

$$
\left\|s_{i}-s\right\|^{2} \geq 2\left\langle u-s, s_{i}-s\right\rangle=2 r^{2} \alpha_{i} \geq 2 r^{2}\left(1-\gamma-\frac{\epsilon_{i}}{2 r}\right)
$$

for $i \in \mathbb{N}$, which implies the statement. 
Remark 8.2 Let $\mathbb{H}$ be a real Hilbert space, $S \subset \mathbb{H}$ be a nonempty subset, $\bar{u} \notin \operatorname{cl} S, \bar{s} \in \operatorname{cl} S$ be given such that $d_{S}(\bar{u})=\|\bar{u}-\bar{s}\|$. Then we have

$$
\forall \epsilon>0, \lim _{t \downarrow 0} \frac{d_{S \cap \mathbb{B}_{\mathbb{H}}[\bar{s}, \epsilon]}(\bar{u}+t(\bar{u}-\bar{s}))-d_{S}(\bar{u})}{t\|\bar{u}-\bar{s}\|} \geq 1-\frac{\epsilon^{2}}{2 d_{S}^{2}(\bar{u})} .
$$

Proof It follows from Lemma 8.1 that

$$
\forall \epsilon>0, \epsilon \geq \sqrt{2\left(1-\lim _{t \downarrow 0} \frac{d_{S \cap \mathbb{B}_{\mathbb{H}}[\bar{s}, \epsilon]}(\bar{u}+t(\bar{u}-\bar{s}))-d_{S \cap \mathbb{B}_{\mathbb{H}}[\bar{s}, \epsilon]}(\bar{u})}{t\|\bar{u}-\bar{s}\|}\right)} d_{S \cap \mathbb{B}_{\mathbb{H}}[\bar{s}, \epsilon]}(\bar{u}),
$$

which implies (35).

Remark 8.3 Let $\mathbb{H}$ be a real Hilbert space, $S \subset \mathbb{H}$ be a nonempty subset, $\bar{u} \notin \operatorname{cl} S, \bar{s} \in \operatorname{cl} S$, $\beta>0$ be given. If $\left\{u_{i}\right\}_{i \in \mathbb{N}}$ is a sequence of elements from $\mathbb{H}$ such that

$$
\lim _{i \longrightarrow \infty} \frac{d_{S}\left(u_{i}\right)-d_{S}(\bar{u})}{\left\|u_{i}-\bar{u}\right\|}=1, \lim _{i \longrightarrow \infty} u_{i}=u \text { and } d_{S}(\bar{u})=\|\bar{u}-\bar{s}\|,
$$

where $u=\bar{u}+\beta(\bar{u}-\bar{s})$, then we have

$$
d_{S}(u)=\|u-\bar{s}\| \text { and } \lim _{t \downarrow 0} \frac{d_{S}\left(\bar{u}+t\|\bar{u}-\bar{s}\|^{-1}(\bar{u}-\bar{s})\right)-d_{S}(\bar{u})}{t}=1 .
$$

Proof Since $\lim _{i \rightarrow \infty} \frac{d_{S}\left(u_{i}\right)-d_{S}(\bar{u})}{\left\|u_{i}-\bar{u}\right\|}=1$, so

$$
\begin{aligned}
d_{S}(u)=\lim _{i \longrightarrow \infty} d_{S}\left(u_{i}\right)=\lim _{i \rightarrow \infty} d_{S}(\bar{u})+\left\|u_{i}-\bar{u}\right\| & =d_{S}(\bar{u})+\|u-\bar{u}\| \\
& =\|\bar{u}-\bar{s}\|+\|u-\bar{u}\|=\|u-\bar{s}\| .
\end{aligned}
$$

It follows from Lemmas 2.6 and 8.1 that $\lim _{t \downarrow 0} \frac{d_{S}\left(\bar{u}+t\|\bar{u}-\bar{s}\|^{-1}(\bar{u}-\bar{s})\right)-d_{S}(\bar{u})}{t}=1$.

Corollary 8.4 Let $\mathbb{H}$ be a real Hilbert space, $S \subset \mathbb{H}$ be a nonempty subset. Then if $u \in$ fr $D_{s}(S), u \neq s$ is such that there is $x \in \operatorname{int} D_{s}(S)$ for which $d_{\mathbb{H} \backslash i n t} D_{s}(S)(x)=\|u-x\|$ then the implication

$$
\lim _{t \downarrow 0} \frac{d_{S}(u+t(u-s))-d_{S}(u)}{t\|u-s\|}<1 \Longrightarrow u \in D_{d}(S) \text { for some } d \in \operatorname{cl} S, d \neq s
$$

holds true.

Proof It follows from Lemma 8.1 that there is a sequence $\left\{s_{i}\right\}_{i \in \mathbb{N}}$ in $S$ such that

$$
\liminf _{i \longrightarrow \infty}\left\|s_{i}-s\right\| \geq \sqrt{2(1-\gamma)} d_{S}(u) \text { and } d_{S}(u)=\lim _{i \longrightarrow \infty}\left\|s_{i}-u\right\| .
$$

By Proposition 3.1 and Lemma 2.8 the sequence $\left\{s_{i}\right\}_{i \in \mathbb{N}}$ has a converging subsequence, say to $d \in S, d \neq s$, and consequently $u \in D_{S}(S) \cap D_{d}(S)$.

Definition 8.5 Let $\mathbb{H}$ be a real Hilbert space. We say that a set $S \subset \mathbb{H}$ is locally approximately weakly compact if for every $u \notin S$ and $\bar{s} \in \operatorname{cl} S$ there is $\delta>0$ such that for every sequence $\left\{s_{i}\right\}_{i \in \mathbb{N}}$ in $\mathbb{B}_{\mathbb{H}}[\bar{s}, \delta] \cap S$ we have the following implication

$$
\left\|s_{i}-u\right\| \longrightarrow d_{S}(u) \text { and } s_{i} \stackrel{\text { weak }}{\longrightarrow} s \in \mathbb{H} \Longrightarrow s \in S .
$$


Corollary 8.6 Let $\mathbb{H}$ be a real Hilbert space, $S \subset \mathbb{H}$ be a nonempty subset and $\bar{s} \in S$, $u \in D_{\bar{s}}(S) \backslash S$ be given such that for every sequence $\left\{s_{i}\right\}_{i \in \mathbb{N}}$ elements of $S$ we have

$$
\left\|s_{i}-u\right\| \longrightarrow d_{S}(u) \text { and } s_{i} \stackrel{\text { weak }}{\longrightarrow} s \in \mathbb{H} \Longrightarrow s \in S .
$$

Then the implication

$$
\lim _{t \downarrow 0} \frac{d_{S}(u+t(u-s))-d_{S}(u)}{t\|u-\bar{s}\|}<1 \Longrightarrow u \in D_{s}(S) \text { for some } s \in S, s \neq \bar{s}
$$

holds true.

Proof It follows from Lemma 8.1 that there is a sequence $\left\{s_{i}\right\}_{i \in \mathbb{N}}$ elements of $S$ such that

$$
\liminf _{i \rightarrow \infty}\left\|s_{i}-\bar{s}\right\| \geq \sqrt{2(1-\gamma)} d_{S}(u) \text { and } d_{S}(u)=\lim _{i \rightarrow \infty}\left\|s_{i}-u\right\| .
$$

Since the sequence $\left\{s_{i}\right\}_{i \in \mathbb{N}}$ is bounded we may suppose that $s_{i} \stackrel{\text { weak }}{\longrightarrow} s \in S$ (if not we choose a proper subsequence). Observe that

$$
\begin{aligned}
& \left\|s_{i}-u\right\|^{2}=\|s-u\|^{2}+2\left\langle s_{i}-s, s-u\right\rangle+\left\|s_{i}-s\right\|^{2} \\
& \quad \Longrightarrow d_{S}(u)=\lim _{i \longrightarrow \infty}\left\|s_{i}-u\right\|^{2} \geq\|s-u\|^{2}+\limsup _{i \longrightarrow \infty}\left\|s_{i}-s\right\|^{2} \\
& \quad \Longrightarrow 0=\lim _{i \longrightarrow \infty}\left\|s_{i}-s\right\|^{2} .
\end{aligned}
$$

Thus

$$
\|s-\bar{s}\|=\liminf _{i \longrightarrow \infty}\left\|s_{i}-s\right\| \geq \sqrt{2(1-\gamma)} d_{S}(u)>0,
$$

and consequently $s \neq \bar{s}, u \in D_{\bar{s}}(S) \cap D_{s}(S)$.

Below a generalization of the V. Klee result is provided in the Hilbert space setting, we refer to [24, Theorem 4.1, page 301] for the Klee result. Namely we show that every Chebyshev set, which is locally approximately weakly compact, is convex.

Theorem 8.7 Let $\mathbb{H}$ be a real Hilbert space, $S \subset \mathbb{H}$ be a nonempty closed and locally approximately weakly compact subset. If $S$ is Chebyshev then it is convex.

Proof Let us fix any $\bar{u} \notin S$. There are $\delta>0, \bar{s} \in S$ such that (36) holds true and

$$
d_{\mathbb{B}_{\mathbb{H}}[\bar{s}, \delta] \cap S}(\bar{u})=d_{S}(\bar{u})=\|\bar{u}-\bar{s}\|>2 \delta .
$$

I. Claim:

$$
\exists \mu \in] 0, \delta\left[: \forall u \in \mathbb{B}_{\mathbb{H}}[\bar{u}, \mu] \cap \operatorname{cl} D_{\mathbb{B}_{\mathbb{H}}[\bar{s}, \delta] \cap S}(S),\left\|P_{\mathbb{B}_{\mathbb{H}}[\bar{s}, \delta] \cap S}(u)-\bar{s}\right\|<\delta .\right.
$$

In fact, if $\left\{u_{i}\right\}_{i \in \mathbb{N}}$ in $\operatorname{cl} D_{\mathbb{B}_{\mathbb{H}}[s, \delta] \cap S}(S)$ is such that $\left\|P_{\mathbb{B}_{\mathbb{H}}[s, \delta] \cap S}\left(u_{i}\right)-\bar{s}\right\| \geq \delta$, for every $i \in \mathbb{N}$ and $P_{\mathbb{B}_{\mathbb{H}}[s, \delta] \cap S}\left(u_{i}\right) \stackrel{\text { weak }}{\longrightarrow} d \in S$ (keep in mind (36)), $u_{i} \longrightarrow \bar{u}$, then

$$
\begin{aligned}
\left\|P_{\mathbb{B}_{\mathbb{H}}[s, \delta] \cap S}\left(u_{i}\right)-\bar{u}\right\|^{2}=\|d-\bar{u}\|^{2}+2\left\langle P_{\mathbb{B}_{\mathbb{H}}[s, \delta] \cap S}\left(u_{i}\right)-d, d-\bar{u}\right\rangle \\
+\left\|P_{\mathbb{B}_{\mathbb{H}}[s, \delta] \cap S}\left(u_{i}\right)-d\right\|^{2} \Longrightarrow d_{S}^{2}(\bar{u})=\lim _{i \longrightarrow \infty}\left\|P_{\mathbb{B}_{\mathbb{H}}[s, \delta] \cap S}\left(u_{i}\right)-\bar{u}\right\|^{2} \\
\geq\|d-\bar{u}\|^{2}+\limsup _{i \longrightarrow \infty}\left\|P_{\mathbb{B}_{\mathbb{H}}[s, \delta] \cap S}\left(u_{i}\right)-d\right\|^{2} \\
\Longrightarrow 0=\lim _{i \longrightarrow \infty}\left\|P_{\left.\mathbb{B}_{\mathbb{H}}[s, \delta] \cap S\right)}\left(u_{i}\right)-d\right\|^{2},
\end{aligned}
$$


so $\|d-\bar{s}\| \geq \delta,\|d-\bar{u}\|=\|\bar{s}-\bar{u}\|$, a contradiction (keep in mind that $S$ is a Chebyshev set). Thus Claim I holds true, and we can choose $\bar{\mu} \in] 0, \delta[$ satisfying (38). Let us observe that for every $u \in \mathbb{B}_{\mathbb{H}}[\bar{u}, \bar{\mu}] \cap D_{\mathbb{B}_{\mathbb{H}}[\bar{s}, \delta] \cap S}(S)$ (19) is satisfied with $P_{\mathbb{B}_{\mathbb{H}}[\bar{s}, \delta] \cap S}(u)$ instead of $\bar{s}$ and $\mathbb{B}_{\mathbb{H}}[\bar{s}, \delta] \cap S$ instead of $S^{\prime}$ and properly chosen $\epsilon>0$ (keep in mind that Claim I is valid), so it follows from Lemma 4.8 that $u \in \operatorname{cl}$ int $D_{\mathbb{B}_{\mathbb{H}}[\bar{s}, \delta] \cap S}(S)$ for every $u \in \mathbb{B}_{\mathbb{H}}[\bar{u}, \bar{\mu}] \cap$ $D_{\mathbb{B}_{\mathbb{H}}[\bar{s}, \delta] \cap S}(S)$.

Claim II:

$$
\lim _{t \downarrow 0} \frac{d_{S}(\bar{u}+t(\bar{u}-\bar{s}))-d_{S}(\bar{u})}{t\|\bar{u}-\bar{s}\|}=1 .
$$

Case I:

$$
\bar{u} \in \operatorname{int} D_{\mathbb{B}_{\mathbb{H}}[\bar{s}, \delta] \cap S}(S) .
$$

In this case $\bar{u}+t(\bar{u}-\bar{s}) \in \operatorname{int} D_{\mathbb{B}_{\mathbb{H}}[\bar{s}, \delta] \cap S}(S)$ for $t>0$ small enough, so $d_{S}(\bar{u}+t(\bar{u}-\bar{s}))=$ $d_{\mathbb{B}_{\mathbb{H}}[\bar{s}, \delta] \cap S}(\bar{u}+t(\bar{u}-\bar{s}))$ for $t>0$ small enough. It follows form Corollary 8.6 that

$$
\lim _{t \downarrow 0} \frac{d_{\mathbb{B}_{\mathbb{H}}[\bar{s}, \delta] \cap S}(\bar{u}+t(\bar{u}-\bar{s}))-d_{\mathbb{B}_{\mathbb{H}}[\bar{s}, \delta] \cap S}(\bar{u})}{t\|\bar{u}-\bar{s}\|}=1,
$$

so Claim II holds true in this case.

Case II:

$$
\bar{u} \notin \operatorname{int} D_{\mathbb{B}_{\mathbb{H}}[\bar{s}, \delta] \cap S}(S) .
$$

It follows from Proposition 4.5 and Claim I that there are $u_{0} \in \operatorname{cl} D_{\mathbb{B}_{\mathbb{H}}[\bar{s}, \delta] \cap S}(S) \backslash$ int $D_{\mathbb{B}_{\mathbb{H}}[\bar{s}, \delta] \cap S}(S)$ is such that $\left\|P_{\mathbb{B}_{\mathbb{H}}[\bar{s}, \delta] \cap S}\left(u_{0}\right)-\bar{s}\right\|<\delta$ and $x_{0} \in$ int $D_{\mathbb{B}_{\mathbb{H}}[\bar{s}, \delta] \cap S}(S)$ such that $\left.d_{\mathbb{H} \backslash \text { int }} D_{\mathbb{B}_{\mathbb{H}}[\bar{s}, \delta] \cap S}(S)=\| x_{0}\right)=\left\|u_{0}-x_{0}\right\|$. Thus we have

$$
\begin{array}{r}
P_{S}\left(u_{0}\right) \cap\left(\mathbb{B}_{\mathbb{H}}[\bar{s}, \delta] \cap S\right)=\left\{P_{\mathbb{B}_{\mathbb{H}}[\bar{s}, \delta] \cap S}\left(u_{0}\right)\right\}, \mathbb{B}_{\mathbb{H}}\left[x_{0},\left\|u_{0}-x_{0}\right\|\right] \subset \operatorname{cl} D_{\mathbb{B}_{\mathbb{H}}[\bar{s}, \delta] \cap S}(S), \\
\lim _{t \longrightarrow 0}|t|^{-1} \sup _{z \in S_{\mathbb{H}}[0,1],\left\langle z, u_{0}-x_{0}\right\rangle=0} d_{D_{\mathbb{B}_{\mathbb{H}}[\bar{s}, \delta] \cap S}(S)}\left(u_{0}+t z\right)=0 .
\end{array}
$$

Since $u_{0} \notin$ int $D_{\mathbb{B}_{\mathbb{H}}}[\bar{s}, \delta] \cap S(S)$, so there are $u_{i} \notin D_{\mathbb{B}_{\mathbb{H}}}[\bar{s}, \delta] \cap S(S)$ such that $u_{i} \longrightarrow u$. For every $i \in \mathbb{N}$ take $d_{i} \in S \backslash \mathbb{B}_{\mathbb{H}}[\bar{s}, \delta] \cap S$ such that $\left.\left\|u_{i}-d_{i}\right\| \longrightarrow \| u_{0}-P_{S}\left(u_{0}\right)\right) \|$. It follows from Proposition 3.1 that there is subsequence $\left\{d_{i_{k}}\right\}_{k \in \mathbb{N}} \subset\left\{d_{i}\right\}_{i \in \mathbb{N}}$ such that $d_{i_{k}} \longrightarrow d^{*} \in S$, $\left\|u_{0}-d^{*}\right\|=\left\|u_{0}-P_{S}\left(u_{0}\right)\right\|$ and $\left\|\bar{s}-d^{*}\right\| \geq \delta$, hence $d^{*} \neq P_{S}\left(u_{0}\right)$ since $\left\|\bar{s}-P_{S}\left(u_{0}\right)\right\|<\delta$, and $u_{0} \in D_{d^{*}}(S) \cap D_{P_{S}\left(u_{0}\right)}(S)$, thus $S$ is not Chebyshev. This contradiction excludes Case II and Claim II holds true, which implies that (22) is satisfied, so by The Vlasov result we get the convexity of $S$.

It is obvious that (36) is satisfied whenever $S$ is weakly closed. Hence the Klee result is an immediate consequence of Theorem 8.7. Because of this, it is of interest which sets are locally approximately weakly compact. Below we indicate some of them in the Hilbert space setting, of course the interested reader can provide examples of such sets in a more general setting. First let us note that if $S=\bigcup_{i \in \mathbb{N}} S_{i}$, where $S_{i}$ are weakly closed and $S \backslash S_{i}$ is closed for every $i \in \mathbb{N}$ then (36) is satisfied. In fact, let us fix $i \in \mathbb{N}$ and $s \in S_{i}$. There is $\epsilon_{i}>0$ such that $\mathbb{B}_{\mathbb{H}}\left[s, \epsilon_{i}\right] \cap S=\mathbb{B}_{\mathbb{H}}\left[s, \epsilon_{i}\right] \cap S_{i}$, hence (36) holds true. It is also easy to notice that if $F: \mathbb{H} \longrightarrow \mathbb{H}$ is a one-to-one operator weakly continuous and $F(S)$ is locally weakly closed subset, then $S$ is locally weakly closed too, so it is locally approximately weakly compact. 
Remark 8.8 Let $\mathbb{H}$ be a real Hilbert space, $S=\left\{s_{1}, s_{2}, \ldots\right\} \subset \mathbb{H}$ be a closed subset such that $s_{i} \stackrel{\text { weak }}{\longrightarrow} s^{*} \in \mathbb{H}$ then

$$
\forall s \in S, \exists \delta>0: \mathbb{B}_{\mathbb{H}}[s, \delta] \cap S=\mathrm{cl}^{\text {weak }}\left(\mathbb{B}_{\mathbb{H}}[s, \delta] \cap S\right),
$$

consequently (36) is satisfied.

Proof If $s^{*} \in S$, then for all $y \notin S$ we have

$$
\lim _{i \longrightarrow \infty}\left\langle y-s^{*}, y-s_{i}\right\rangle=\left\|y-s^{*}\right\|^{2}>0,
$$

so $y$ is not in the weak closure of $S$, hence $S$ is weakly closed and we are done. If $s^{*} \notin S$ then

$$
\forall s \in S, \exists \delta>0: s^{*} \notin \mathbb{B}_{\mathbb{H}}[s, \delta],
$$

so $\mathbb{B}_{\mathbb{H}}[s, \delta] \cap S=\mathrm{cl}^{\text {weak }}\left(\mathbb{B}_{\mathbb{H}}[s, \delta] \cap S\right)$, since if $s^{\prime} \in \mathrm{cl}^{\text {weak }} \mathbb{B}_{\mathbb{H}}[s, \delta] \cap S \backslash S$, then $s^{\prime}=s^{*}$ but $\left\|s^{\prime}-s\right\|<\delta$ and $\left\|s^{*}-s\right\| \geq \delta$, a contradiction.

A direct consequence of Lemma 2.6 is

Remark 8.9 Let $\mathbb{H}$ be a real Hilbert space, $S \subset \mathbb{H}$ be a nonempty closed subset such that

$$
\forall u \notin S, \forall \bar{s} \in P_{S}(u), \exists \delta>0: \lim _{t \downarrow 0} \frac{d_{S \cap B_{\mathbb{H}}[\bar{s}, \delta]}(u+t(u-\bar{s}))-d_{S}(u)}{t\|u-\bar{s}\|}=1
$$

then

$$
\forall u \notin S, \forall \bar{s} \in P_{S}(u), \exists \delta>0:\left\|\bar{s}_{i}-u\right\| \longrightarrow d_{S}(u) \Longrightarrow s \in S,
$$

whenever $\left\{\bar{s}_{i}\right\}_{i \in \mathbb{N}}$ is in $\mathbb{B}_{\mathbb{H}}[\bar{s}, \delta] \cap S$, and consequently (36) is satisfied.

Below it is stated that if $S=\bigcup_{i \in \mathbb{N}} S_{i}$, where $S_{i}$ are closed convex nonempty subsets, then $S$ can not be Chebyshev set, whenever it is not convex.

Theorem 8.10 Let $\mathbb{H}$ be a real Hilbert space, $S$ be a closed nonempty subset such that $\mathbb{H} \backslash S \subset \bigcup_{i \in \mathbb{N}} D_{S_{i}}(S)$, where $S_{i} \subset S$ are closed convex nonempty subsets. If $S$ is not convex, then there are $d_{1}, d_{2} \in S, d_{1} \neq d_{2}$ such that $D_{d_{1}}(S) \cap D_{d_{2}}(S) \cap(\mathbb{H} \backslash S) \neq \emptyset$.

Proof Let us suppose that for every $d_{1}, d_{2} \in S, d_{1} \neq d_{2}$ we have $D_{d_{1}}(S) \cap D_{d_{2}}(S) \cap$ $(\mathbb{H} \backslash S)=\emptyset$, otherwise we are done. By by the Baire Category Theorem, see [37, Baire's Category Argument, The Baire-Hausdorff Theorem, page 11] there is $i_{1} \in \mathbb{N}$ such that

$$
\text { int } D_{S_{i_{1}}}(S) \cap(\mathbb{H} \backslash S) \neq \emptyset \text {. }
$$

Observe that $\left(\operatorname{cl} D_{S_{i_{1}}}(S) \backslash \operatorname{int} D_{S_{i_{1}}}(S)\right) \cap(\mathbb{H} \backslash S)=\emptyset$. If not then for every $u \in$ cl int $D_{S_{i_{1}}}(S) \cap(\mathbb{H} \backslash S)$ such that $] P_{S_{i_{1}}}(u), u\left[\subset \operatorname{int} D_{S_{i_{1}}}(S)\right.$ and $\mathbb{B}_{\mathbb{H}}[x,\|u-x\|] \subset D_{S_{i_{1}}}(S)$ for some $x \in$ int $D_{S_{i_{1}}}(S)$, it follows Corollary 5.1 that there is $d \in S \backslash\left\{P_{S_{i_{1}}}(u)\right\}$ such that $u \in D_{d}(S)$, which contradicts our supposition. Hence $\left(\operatorname{cl} D_{S_{i_{1}}}(S) \backslash \operatorname{int} D_{S_{i_{1}}}(S)\right) \cap(\mathbb{H} \backslash S)=$ $\emptyset$ and consequently

$$
\begin{array}{r}
\forall x^{\prime} \in \operatorname{int} D_{S_{i_{1}}}(S) \backslash S_{i_{1}}, \bigcup_{t \in] 0, \infty[} B\left[P_{S_{i_{1}}}\left(x^{\prime}\right)+t\left(x^{\prime}-P_{S_{i_{1}}}\left(x^{\prime}\right)\right), t\left\|x^{\prime}-P_{S_{i_{1}}}\left(x^{\prime}\right)\right\|\right] \\
\cap S=\left\{P_{S_{i_{1}}}\left(x^{\prime}\right)\right\} .
\end{array}
$$


It is easy to observe that if

$$
\operatorname{int}\left(\mathbb{H} \backslash\left(\operatorname{int} D_{S_{i_{1}}}(S) \cup S\right)\right)=\emptyset,
$$

then

$$
(\mathbb{H} \backslash S) \subset D_{S_{i_{1}}}(S),
$$

so $d_{S}(u)=d_{S_{i_{1}}}(u)$ for every $u \in \mathbb{H} \backslash S$. Hence it follows from Theorem 6.1 that $S$ is convex, a contradiction. Suppose that

$$
\operatorname{int}\left(\mathbb{H} \backslash\left(\operatorname{int} D_{S_{i_{1}}}(S) \cup S\right)\right) \neq \emptyset \text {. }
$$

Again by the Baire Theorem choose $i_{2} \in \mathbb{N} \backslash\left\{i_{1}\right\}$ such that

$$
\text { int } D_{S_{i_{2}}}(S) \cap\left(\mathbb{H} \backslash\left(\text { int } D_{S_{i_{1}}}(S) \cup S\right)\right) \neq \emptyset \text {. }
$$

By a similar reasoning as above we get

$$
\begin{array}{r}
\forall x^{\prime} \in \operatorname{int} D_{S_{i_{2}}}(S) \backslash S_{i_{2}}, \bigcup_{t \in] 0, \infty[} B\left[P_{S_{i_{2}}}\left(x^{\prime}\right)+t\left(x^{\prime}-P_{S_{i_{2}}}\left(x^{\prime}\right)\right), t\left\|x^{\prime}-P_{S_{i_{2}}}\left(x^{\prime}\right)\right\|\right] \\
\cap S=\left\{P_{S_{i_{2}}}\left(x^{\prime}\right)\right\} .
\end{array}
$$

If

$$
\operatorname{int}\left(\mathbb{H} \backslash\left(\operatorname{int} D_{S_{i_{1}}}(S) \cup \operatorname{int} D_{S_{i_{2}}}(S) \cup S\right)\right)=\emptyset \text {, }
$$

then

$$
(\mathbb{H} \backslash S) \subset D_{S_{i_{1}}}(S) \cup D_{S_{i_{2}}}(S) .
$$

Hence it follows from Theorem 6.1 that $S$ is convex, a contradiction.

By the Kuratowski-Zorn Lemma we find $I \subset \mathbb{N}$ such that $j_{1} \neq j_{2}$, whenever $j_{1}, j_{2} \in I$; and int $D_{S_{j}}(S) \cap(\mathbb{H} \backslash S) \neq \emptyset$ and

$\forall x^{\prime} \in \operatorname{int} D_{S_{j}}(S) \backslash S_{j}, \bigcup_{t \in] 0, \infty[} B\left[P_{s_{j}}\left(x^{\prime}\right)+t\left(x^{\prime}-P_{s_{j}}\left(x^{\prime}\right)\right), t\left\|x^{\prime}-P_{s_{j}}\left(x^{\prime}\right)\right\|\right] \cap S=\left\{P_{s_{j}}\left(x^{\prime}\right)\right\}$

for every $j \in \mathbb{N}$, and int $\left(\mathbb{H} \backslash\left(S \cup \bigcup_{s \in I}\right.\right.$ int $\left.\left.D_{S_{i}}(S)\right)\right)=\emptyset$. It follows from Corollary 6.2 that (22) holds true for all $x \notin S$, and by the L.P. Vlasov results the set $S$ is convex, but this is impossible.

Theorem 8.10 when compared with [2, Theorem 2.19] has the following differences: first, it is given in a Hilbert space, while [2, Theorem2.19] is given in a more general space, namely in the smooth Efimov-Stechkin space; second, it is not assumed that its boundary is included in a countable union of hyperplanes, as it was done in [2, Theorem]. So, it is natural to expect a result combining advantages of both theorems, but this is not the aim of this paper.

Let us recall the following problem raised by K. Goebel and R. Schöneberg: Does there exist a convex body $Y \subset \mathbb{H}$ such that the boundary of $Y$ is a Chebyshev set with respect to its convex closure, in other words is $S:=\mathbb{H} \backslash$ int $Y$ a Chebyshev set for some convex bounded set $Y \subset \mathbb{H}$ having nonempty interior, see [17, Problem 1, page 466]? A. P. Bosznay gave the answer in the negative the question, whenever the boundary of $Y$ is included in a countable union of hyperplanes, see [4, Theorem, page 143], see also [2, Theorem 2.19], where a generalization of this result was given and several results on the convexity of Chebyshev sets can be also found. In view of Corollary 8.11 it is enough to know that a part of the boundary is flat in order to answer the question in the negative, the details are presented 
in the Corollaries below. First, is shown that condition (14) can be used to have $S$ in some halfspace.

Corollary 8.11 Let $\mathbb{H}$ be a real Hilbert space, $S \subset \mathbb{H}$ be a nonempty closed subset, $\bar{s} \in S$, $u \notin S, \epsilon>0$ be given such that $d_{S}(u)=\|u-\bar{s}\|$ and $B_{\mathbb{H}}[\bar{s}, \epsilon] \cap\{h \in \mathbb{H} \mid\langle u-\bar{s}, h-\bar{s}\rangle=$ $0\} \subset S$, and

$$
\begin{aligned}
\forall s^{\prime} \in B_{\mathbb{H}}[\bar{s}, \epsilon] \cap S,\left\langle u-\bar{s}, s^{\prime}-\bar{s}\right\rangle \leq & 0 \text { and } P_{S}\left(D_{B_{\mathbb{H}}[\bar{s}, \epsilon] \cap\{h \in \mathbb{H} \mid\langle u-\bar{s}, h-\bar{s}\rangle=0\}}(S)\right) \\
& \subset B_{\mathbb{H}}[\bar{s}, \epsilon] \cap\{h \in \mathbb{H} \mid\langle u-\bar{s}, h-\bar{s}\rangle=0\} .
\end{aligned}
$$

Then $\{\bar{s}\}=P_{S}(u) \cap B_{\mathbb{H}}[\bar{s}, \epsilon] \cap S$ and

$$
\left\|s_{i}-u\right\| \longrightarrow d_{S}(u) \Longrightarrow s_{i} \longrightarrow \bar{s},
$$

for every sequence $\left\{s_{i}\right\}_{i \in \mathbb{N}}$ in $S$, and

$$
\forall s \in S,\langle u-\bar{s}, s-\bar{s}\rangle \leq 0 .
$$

Proof Put $S^{\prime}:=B_{\mathbb{H}}[\bar{s}, \epsilon] \cap\{h \in \mathbb{H} \mid\langle u-\bar{s}, h-\bar{s}\rangle=0\}$. It follows from Corollary 4.7 that $u \in \operatorname{cl}$ int $D_{\mathbb{B}_{\mathbb{H}}}[\bar{s}, \epsilon] \cap S(S)$. If $u \in$ int $D_{S^{\prime}}(S)$ then (42) is a consequence of Lemma 2.4. Let us consider the case $u \in \operatorname{cl}$ int $D_{S^{\prime}}(S) \backslash$ int $D_{S^{\prime}}(S)$. It follows from Corollary 5.5 that

$$
\left.u \in(\mathbb{H} \backslash S) \cap\left(\operatorname{clint} D_{S^{\prime}}(S) \backslash\left(\operatorname{int} D_{S^{\prime}}(S) \cup S^{\prime}\right)\right)\right) \subset \operatorname{cl}\left(\bigcup_{d \in \operatorname{cl}\left(S \backslash S^{\prime}\right)} D_{d}(S) \cap D_{S^{\prime}}(S)\right) .
$$

However, the inclusion above is not valid, since it contradicts to (41). Indeed, if $u_{i} \longrightarrow u$, $d_{i} \in \operatorname{cl}\left(S \backslash S^{\prime}\right), u_{i} \in D_{d_{i}}(S) \cap D_{S^{\prime}}(S)$ for every $i \in \mathbb{N}$, then by the inclusion in (41) we get $d_{i} \in S^{\prime}$ for every $i \in \mathbb{N}$. Observe that $d_{i} \in \operatorname{cl}\left(S \backslash S^{\prime}\right)$ and $d_{i} \in S^{\prime}$ imply $\left\|d_{i}-\bar{s}\right\|=\epsilon$, which contradicts the continuity of $P_{S^{\prime}}$, that is we should have had $d_{i}=P_{S^{\prime}}\left(u_{i}\right) \longrightarrow \bar{s}$ since $u_{i} \longrightarrow u$. Thus $u+t(u-\bar{s}) \in \operatorname{int} D_{S^{\prime}}(S)$ for every $t>0$, otherwise we repeat the reasoning above to get a contradiction with $u_{\bar{t}}:=u+\bar{t}(u-\bar{s})$, where $\bar{t}>0$ is such that $u+\bar{t}(u-\bar{s}) \in \operatorname{cl}$ int $D_{S^{\prime}}(S) \backslash$ int $D_{S^{\prime}}(S)$. The condition $u+t(u-\bar{s}) \in \operatorname{int} D_{S^{\prime}}(S)$ for every $t>0$, implies (43).

Corollary 8.12 Let $\mathbb{H}$ be a real Hilbert space, $U \subset \mathbb{H}$ be an open nonempty and convex subset, and $S:=\mathbb{H} \backslash U$ be a nonempty Chebyshev subset such that for some $\bar{s} \in S, u \in U$, $\epsilon>0$ we have $d_{S}(u)=\|u-\bar{s}\|$ and $B_{\mathbb{H}}[\bar{s}, \epsilon] \cap\{h \in \mathbb{H} \mid\langle u-\bar{s}, h-\bar{s}\rangle=0\} \subset S$, and

$$
\forall s^{\prime} \in B_{\mathbb{H}}[\bar{s}, \epsilon] \cap S,\left\langle u-\bar{s}, s^{\prime}-\bar{s}\right\rangle \leq 0 .
$$

Then $U=\{h \in \mathbb{H} \mid\langle u-\bar{s}, h-\bar{s}\rangle>0\}$.

Proof Since $S$ is a Chebyshev set we have

$$
P_{S}\left(D_{B_{\mathbb{H}}[\bar{s}, \epsilon] \cap\{h \in \mathbb{H} \mid\langle u-\bar{s}, h-\bar{s}\rangle=0\}}(S)\right) \subset B_{\mathbb{H}}[\bar{s}, \epsilon] \cap\{h \in \mathbb{H} \mid\langle u-\bar{s}, h-\bar{s}\rangle=0\},
$$

hence by Corollary 8.11 we get

$$
\forall s \in S,\langle u-\bar{s}, s-\bar{s}\rangle \leq 0,
$$

which implies the inclusion $\{h \in \mathbb{H} \mid\langle u-\bar{s}, h-\bar{s}\rangle>0\} \subset U$. The convexity of $U$ and (44) ensure the reverse inclusion $U \subset\{h \in \mathbb{H} \mid\langle u-\bar{s}, h-\bar{s}\rangle>0\}$. 
Open Access This article is distributed under the terms of the Creative Commons Attribution 4.0 International License (http://creativecommons.org/licenses/by/4.0/), which permits unrestricted use, distribution, and reproduction in any medium, provided you give appropriate credit to the original author(s) and the source, provide a link to the Creative Commons license, and indicate if changes were made.

\section{References}

1. Asplund, E.: Čebyšev sets in Hilbert spaces. Trans. Amer. Math. Soc. 144, 235-240 (1969)

2. Balaganskii, V.S., Vlasov, L.P.: The problem of convexity of Chebyshev sets. Russ. Math. Surv. 51(6), 1127-1190 (1996)

3. Bernard, F., Thibault, L.: Prox-regular functions in Hilbert spaces. J. Math. Anal. Appl. 303, 1-14 (2005)

4. Bosznay, A.P.: A remark on a problem of Goebel. Ann. Univ. Set. Budapest Eotvos Sect. Math. 28, 143145 (1985)

5. Borwein, J.M., Fitzpatrick, S.P., Giles, J.R.: The Differentiability of real functions on normed linear space using generalized subgradients. J. Math. Anal. Appl. 128, 512-534 (1987)

6. Borwein, J.M., Zhu, Q.J.: Techniques of Variational Analysis. Springer (2005)

7. Borwein, J.M.: Proximality and Chebyshev sets. Optimization letters, 21-32 (2007)

8. Correa, R., Jofré, A., Thibault, L.: Characterization of Lower Semicontinuous Convex Functions. Proc. Amer. Math. Soc. 116(1), 67-72 (1992)

9. Deville, R., Godefroy, G., Zizler, V.: Smoothness and renormings in Banach spaces. Longman Scintific and Technical (1993)

10. Diestel, J.: Geometry of Banach spaces-selected topics, Springer- Verlag Lecture Notes 485 (1975)

11. Dutta, S.: Generalized Subdifferential of the distance function. Proc. Amer. Math. Soc., 133(10), 29492955 (2005)

12. Fabian, M., Habala, P., Hajek, P., Montesinos, V., Zizler, V.: Banach Space Theory, The Basis for Linear and Nonlinear Analysis. Springer, New York (2011)

13. Fitzpatrick, S.: Metric projections and the differentiability of distance functions. Bull. Austral. Math. Soc. 22, 291-312 (1980)

14. Fitzpatrick, S.: Differentiation of real-valued functions and continuity of metric projections. Proc. Amer. Math. Soc. 91(4), 544-548 (1984)

15. Fitzpatrick, S.: Nearest points to closed sets and directional derivatives of distance functions. Bull. Austral. Math. Soc. 39, 233-238 (1989)

16. Friedman, A.: Foundations of Modern Analysis. Dover Publications Inc, New York (1982)

17. Goebel, K., Schöneberg, R.: Moons, bridges, birds... and nonexpasive mappings in Hilbert space. Bull. Austral. Math. Soc. 17, 463-466 (1977)

18. Giles, J.R.: Differentiability of distance functions and a proximal property inducing convexity. Proc. Amer. Math. Soc. 104, 458-464 (1988)

19. Giles, J.R.: A distance function property implying differentiability. Bull. Austral. Math. Soc. 39, 59-70 (1989)

20. Hiriart-Urruty, J.-B.: New concepts in nondifferentiable programming. Mémoires de la S.M.F., tome 60, 57-85 (1979)

21. Hiriart-Urruty, J.-B.: Ensembles de Tchebychev vs. ensembles convexes: Létat de lart vu via lanalyse convexe non lisse. Ann. Sci. Math. Québec 22, 4762 (1998)

22. Hiriart-Urruty, J.-B.: Potpourri of Conjectures and Open Questions in Nonlinear Analysis and Optimization. SIAM Review 49, 255-273 (2007)

23. Jourani, A., Thibault, L., Zagrodny, D.: Differential properties of the Moreau envelope. J. Fun. Anal. 266, 1185-1237 (2014)

24. Klee, V.: Convexity of Chebyshev sets. Math. Annalen 142, 292-304 (1961)

25. Klee, V.L.: Remarks on nearest points in normed linear spaces, in Proc. Colloquium on Convexity (Copenhagen 1965), 168176, Kobenhavns Univ. Mat. Inst., Copenhagen (1967)

26. Klee, V.L.: Reproduced with comments by B. Grübbaum, Unsolved problems in intuitive geometry. http://www.math.washington.edu/ (1960/2010)

27. Lau, K.-S.: Almost Chebyshev Subspaces. J. Approx. Theory 21, 319-327 (1977)

28. Lau, K.-S.: On almost Chebyshev subsets in reflexive Banach spaces. Indiana Univ. Math. J. 27, 791-795 (1978)

29. Poliquin, R.A., Rockafellar, R.T., Thibault, L.: Local differentiability of distance functions. Trans. Amer. Math. Soc. 352, 5231-5249 (2000) 
30. Ricceri, B.: A conjecture implying the existence of non-convex Chebyshev sets in infinite-dimensional Hilbert spaces. Le Matematiche LXV, 193-199 (2010). - Fasc. II

31. Rudin, W.: Functional Analysis. McGraw-Hill, New York (1973)

32. Westphal, U., Frerking, J.: On a property of metric projections onto closed subsets of Hilbert spaces. Proc. Amer. Math. Soc. 105, 644-651 (1989)

33. Vlasov, L.P.: Chebyshev sets and approximatively convex sets. Math. Notes 2, 600-605 (1967)

34. Vlasov, L.P.: Almost convexity and Chebyshev sets. Math. Notes 8, 776-779 (1970)

35. Vlasov, L.P.: Approximative properties of sets in normed linear spaces. Russian Math. Surveys 28(174), 3-66 (1973)

36. Wang, X.: On Chebyshev functions and Klee functions. J. Math. Anal. Appl. 368, 293-310 (2010)

37. Yosida, K. Functional Analysis, 6th Edn. Springer, Berlin (1980)

38. Zhivkov, N.V.: Metric projections and antiprojections in strictly convex normed spaces. C.R. Acad. Bulgare Sci. 31(4), 369-372 (1978) 